\title{
Dynamic Network Design Problem under Demand Uncertainty: An Adjustable Robust Optimization Approach
}

\author{
Hua Sun, ${ }^{1}$ Ziyou Gao, ${ }^{2}$ and Fangxia Zhao ${ }^{1}$ \\ ${ }^{1}$ MOE Key Laboratory for Urban Transportation Complex Systems Theory and Technology, Beijing Jiaotong University, \\ Beijing 100044, China \\ ${ }^{2}$ Institute of System Science, School of Traffic and Transportation, Beijing Jiaotong University, Beijing 100044, China
}

Correspondence should be addressed to Hua Sun; sunhua19800601@163.com

Received 31 October 2013; Accepted 18 December 2013; Published 17 February 2014

Academic Editor: Huimin Niu

Copyright (C) 2014 Hua Sun et al. This is an open access article distributed under the Creative Commons Attribution License, which permits unrestricted use, distribution, and reproduction in any medium, provided the original work is properly cited.

\begin{abstract}
This paper develops an adjustable robust optimization approach for a network design problem explicitly incorporating traffic dynamics and demand uncertainty. In particular, a cell transmission model based network design problem of linear programming type is considered to describe dynamic traffic flows, and a polyhedral uncertainty set is used to characterize the demand uncertainty. The major contribution of this paper is to formulate such an adjustable robust network design problem as a tractable linear programming model and justify the model which is less conservative by comparing its solution performance with the robust solution from the usual robust model. The numerical results using one network from the literature demonstrate the modeling advantage of the adjustable robust optimization and provided strategic managerial insights for enacting capacity expansion policies under demand uncertainty.
\end{abstract}

\section{Introduction}

Over the past three decades, network design problem (NDP) has been pervasively studied and applied in the different fields, such as transportation, production, distribution, and communication. The different research field is corresponding to different sets of objectives, decision variables, and resource constraints, implying different behavioral and system assumptions, and possessing varying parameters and data requirements and capabilities in terms of representing the network supplies and demands [1]. NDP models have been extensively used as a decision-making tool and provide the guidelines for enacting the capacity expansion policies. In transportation, network design models in general aim at maximizing the social benefit (or minimizing the total travel costs) through implementing an optimal capacity expansion policy in the network.

However, traditional NDP models for transportation networks assume that the demand or/and capacity are deterministic. Obviously, this is unrealistic, because the real-life transportation network systems are surrounded by a number of uncertainties, both from the supply side and demand side.
Evaluation of network performance without accounting for demand uncertainty can potentially lead to biased investment decisions [2]. Therefore, recently, NDPs under uncertainty have drawn increasing attentions. Chen et al. [3] provided a state-of-the-art review of the transportation network design problem under uncertainty.

However, the vast body of the literature in the past has focused on the static NDPs [4-6]. Lin et al. [7] pointed out the three drawbacks of the static NDP models when compared with the dynamic NDP (DNDP) models. Therefore, to overcome these deficiencies, a variety of recent papers have focused on dynamic NDP models [7-11]. In dynamic NDP models, dynamic traffic assignment (DTA) models are used to model the time-dependent variation of traffic flows and travel behaviors and characterize the transportation network flow pattern. Janson [12] and Waller [13] showed that the DTA-based NDP model is more desirable than the static model. According to their formulations, DTA-based NDP models can be classified into two categories: single-level models and bilevel models [7]. The focus of this paper is on the application adjustable robust optimization (ARO) for 
the single-level dynamic NDP under demand uncertainty, where the single-level structure is adopted because it can provide an easier way to manipulate affinely adjustable robust counterpart (AARC) and make adjustable robust dynamic NDP (ARDNDP) to be computationally tractable.

There is an increasing body of relevant literature on the DTA-based NDP under uncertainty [1, 2, 10, 14-18]. The common feature of these studies is that the cell transmission model (CTM) $[19,20]$ is adopted to model the time-varying traffic flows propagation, and the traffic demand is assigned to the network by either the dynamic system-optimal (SO) [21] or user-optimal (UO) [10] principle. The approaches of addressing uncertainty in above-mentioned studies mainly are stochastic programming (SP) and robust optimization (RO). However, these approaches suffer from deficiencies related to lack of data availability or are overly conservative, which seriously limit their applicability in traffic management and control.

In this paper, we employ the ARO approach to develop the single-level CTM-based system-optimal NDP model. Given the fact that the single-level CTM-based NDP model has a linear programming formulation, we use the ARO method [22] to formulate the ARDNDP, which overcomes the limitation of stochastic optimization and usual robust optimization [23-27]. Specifically, not only is there no such assumption that the probability distribution of uncertain demand is known but also it is less conservative than robust dynamic NDP in our model. Similar to robust optimization approach, we only need to simply specify a closed set to limit the demand uncertainty, which is readily implemented in most applications. The adjustable robust counterpart (ARC) of system-optimal DNDP (SODNDP) can be reformulated as the computationally tractable affinely adjustable robust counterpart (AARC) of SODNDP by using prescribed uncertainty set and the affine decision rule.

We emphasize the main contributions of this paper at a glance below.

We provide an ARO framework for the SO DTA-based ARDNDP. Note that we describe the ARDNDP model only for single-destination, system-optimal networks. However, this is unrealistic. How to extend the basic ARCC formulation method to the multidestination problem case is our future research direction.

An appealing feature of our AARC is that it is less conservative than the usual RC. Moreover, AARC is still a linear programming model. So, in general, it is computationally tractable and can be solved in polynomial time by a few wellknown solution algorithms.

Our numerical example demonstrates the value of ARO in the context of dynamic NDP. The computation viability is demonstrated for the proposed modeling framework. The numerical analysis for the impact of investment budget bound and the demand uncertainty level on the network design solution justifies the solution robustness and flexibility.

The remaining part of this paper is structured as follows. Section 2 provides a discussion of the relevant literature. In Section 3, we describe the formulation given by [21] as a CTM-based deterministic dynamic NDP. Section 4 proposes an affinely adjustable robust counterpart formulation of the dynamic NDP under demand uncertainty by using the affine decision rule. In Section 5, we develop a traditional robust counterpart formulation of the dynamic NDP under demand uncertainty. Numerical experiments and results analysis are elaborated in Section 6. Finally, Section 7 concludes the paper and proposes the potential future extensions.

\section{Literature Review}

Dynamic NDP is at the core of many transportation planning problems and has been extensively studied in the literature. Although a great amount of attention has been paid to the deterministic dynamic NDP in the past years, increasing efforts are focusing on incorporating uncertainty in the dynamic NDP because the researchers have realized that input data and parameters have the inherently uncertain nature and uncertain input data and parameters may have a drastic impact on the optimality and the feasibility of the solution. Therefore, various modeling techniques are applied to cope with uncertainty, which mainly include MonteCarlo sampling approach, chance-constrained programming (CCP), a two-stage stochastic programming with recourse (SLP2), scenario-based robust optimization, and set-based robust optimization. Karoonsoontawong and Waller [15] applied Monte-Carlo bounding techniques to solve the stochastic SO and UO dynamic NDP. They showed that it is more beneficial to solve a stochastic model than a deterministic model. Waller and Ziliaskopoulos [14] formulated the CCP and SLP2 models of single-level CTM-based SO NDP under demand uncertainty. Ukkusuri and Waller [10] introduced the CTM-based single-level UO versions of CCP and SLP2 and compared them with the corresponding SO versions. Karoonsoontawong and Waller [17] proposed a SLP2 model of CTM-based bilevel NDP. Karoonsoontawong and Waller [18] further developed a SLP2 model of CTMbased bilevel combined NDP and signal setting design (SSD). The above-mentioned CTM-based NDP models were, however, developed by the Monte-Carlo sampling, CCP, or SLP2 approach and it is necessary for the modeler users to know the probability distributions of the uncertain input data and parameters in order to use these models. In fact, the distributions may be unavailable (inaccurate) in reality because we may have no (insufficient) data to calibrate the distributions. Therefore, robust optimization was introduced recently to address the limitations of CTM-based NDPs or DTA. According to Chung et al. [1], robust optimization can be roughly classified into two groups: (1) scenario-based robust optimization and (2) set-based robust optimization.

Mulvey et al. [28] developed a scenario-based RO approach for general linear programming. Karoonsoontawong and Waller [16] used this approach to propose the CTM-based bilevel NDP model with exact solution methods and developed three metaheuristics for multiorigin multidestination large problem: simulated annealing, genetic algorithm, and random search. Karoonsoontawong and Waller [17] adopted the same approach to formulate CTM-based single-level SO and UO versions of NDP models and bilevel 
NDP model and compared them. Karoonsoontawong and Waller [18] presented a bilevel scenario-based robust model for the CTM-based combined NDP and SSD. Similar to Monte-Carlo sampling, CCP, and SLP2, the scenario-based RO approach also need to know the probability of each scenario in advance. In additional, the numerous scenarios used in accurately representing the uncertainty can lead to large, computationally challenging problems and the solution obtained may be sensitive to possible uncertainty outcomes.

To overcome the limitation of Monte-Carlo sampling, CCP, SLP2, and scenario-based RO approaches, the set-based RO approach has been applied to cope with uncertain input data and parameters. Unlike Monte-Carlo sampling, CCP, SLP2, and scenario-based RO approaches, the set-based RO approach [23-27] does not require the assumption that the probability distributions of the uncertain input data and parameters are known, while the uncertain input data and parameters are assumed to belong to a bounded and convex set. Therefore, recently, the set-based RO has been applied to not only static NDP [29-31] but also CTM-based dynamic NDP or DTA. In CTM-based dynamic NDP or DTA, Chung et al. [1] applied the set-based RO approach to formulate a single-level robust NDP model under demand uncertainty, in which the demand uncertainty is assumed to belong to a box set. Yao et al. [32] used the polyhedral, box, and ellipsoid as uncertain set to develop the CTM-based systemoptimal DTA (SODTA). Chung et al. [33] used the setbased $\mathrm{RO}$ approach to develop dynamic congestion pricing under dynamic user equilibrium. They proposed a bilevel cellular particle swarm optimization (BSCPO) algorithm to solve the corresponding robust counterpart and compared it with bilevel simulated annealing (BSA) and cutting planebased simulated annealing (CPSA) algorithms. They showed that the BSCPO algorithm proposed outperforms these two alternative algorithms through test results. However, the robust solutions obtained in the above set-based RO studies are overly conservative. Therefore, Ben-Tal proposed an adjustable robust optimization formulation (ARO) for general linear programming that can alleviate the conservatism of robust solutions obtained through the usual RO approach. Ben-Tal et al. [34] applied the ARO methodology to solve the CTM-based SODTA under demand uncertainty. The polyhedral set is adopted as the uncertain demand set, and the affinely adjustable robust counterpart (AARC) was reformulated as linear programming by using the affine decision rule and duality theory. In this paper, we extend ARO approach to the CTM-based SO NDP model and provide guidance for making capacity expansion plans under uncertainty in context of transportation network design problem.

\section{Deterministic Model}

In this section, we present the deterministic version of the CTM-based single-level SO dynamic NDP model, which provides the basic modeling platform and functional form the ARDNDP model we will introduce in the next section. For discussion convenience, we present the notation used throughout these models (see Notations).
The CTM-based single-level SO dynamic DNP (SODNDP) model aims at minimizing the total travel cost, which is the sum of the product of the number of vehicles in each cell in each time interval and the corresponding travel cost. To penalty unmet demand by the end of time horizon $T$, the travel cost in cell $i$ at time $t, c_{i}^{t}$, is set as follows:

$$
c_{i}^{t}= \begin{cases}1 & i \in C \backslash C_{s}, t \neq T \\ M & i \in C \backslash C_{s}, t=T,\end{cases}
$$

where $M$ is a sufficiently large positive number to represent the penalty cost, which can be interpreted as the cost of a vehicle that cannot arrive at the destination by the end of time horizon $T$. Use of the penalty cost has the effect of minimizing the number of vehicles staying in the network by the end of the time horizon $T$. By assuming SO principle and the linear relationship between investment and capacity increase and using the notation in Notations, the deterministic CTMbased single-level SODNDP can be formulated as the following linear programming [9]:

SODNDP:

$$
\begin{array}{ll}
\min _{x, y, b} & \sum_{t \in \mathfrak{I}} \sum_{i \in C \backslash C_{s}} c_{i}^{t} x_{i}^{t} \\
\text { subject to } \quad x_{i}^{t}-x_{i}^{t-1}-\sum_{k \in C} a_{k i} y_{k i}^{t-1}+\sum_{j \in C} a_{i j} y_{i j}^{t-1}=d_{i}^{t-1}, \\
\forall i \in C_{R}, t \in \mathfrak{J},
\end{array}
$$

$$
\begin{array}{r}
x_{i}^{t}-x_{i}^{t-1}-\sum_{k \in C} a_{k i} y_{k i}^{t-1}+\sum_{j \in C} a_{i j} y_{i j}^{t-1}=0, \\
\forall i \in C \backslash C_{R} \cup C_{S}, t \in \mathfrak{\Im},
\end{array}
$$

$$
\begin{gathered}
\sum_{j \in C} a_{i j} y_{i j}^{t} \leq x_{i}^{t}, \quad \forall i \in C \backslash C_{S}, t \in \mathfrak{J}, \\
\sum_{k \in C} a_{k i} y_{k i}^{t} \leq \delta_{i}^{t}\left(N_{i}^{t}+\chi_{i} b_{i}-x_{i}^{t}\right), \\
\forall i \in C \backslash C_{R} \cup C_{S}, t \in \mathfrak{I}, \\
\sum_{k \in C} a_{k i} y_{k i}^{t} \leq Q_{i}^{t}+\phi_{i} b_{i}, \\
\forall i \in C \backslash C_{R} \cup C_{S}, t \in \mathfrak{J}, \\
\sum_{j \in C} a_{i j} y_{i j}^{t} \leq Q_{i}^{t}+\phi_{i} b_{i}, \\
\forall i \in C \backslash C_{S}, t \in \mathfrak{I}, \\
x_{i}^{0}=\hat{x}_{i}, \quad \forall i \in C \backslash C_{S}, \\
y_{i j}^{0}=0, \quad \forall(i, j) \in C \times C, \\
x_{i}^{t} \geq 0, \quad \forall i \in C \backslash C_{S}, t \in \mathfrak{I},
\end{gathered}
$$




$$
\begin{aligned}
& y_{i j}^{t} \geq 0, \quad \forall(i, j) \in C \times C, t \in \mathfrak{J}, \\
& b_{i} \geq 0, \quad \forall i \in C \backslash C_{S}, \\
& \sum_{i \in C \backslash C_{s}} b_{i} \leq B .
\end{aligned}
$$

The objective function of SONDP presents the total travel cost, which provides an optimistic estimate or lower bound of total cost as it simplifies the original CTM model by Daganzo $[19,20]$ and allows vehicle holding [35]. Both constraints (3) and (4) are the flow conservation constraints in cell $i$ at time interval $t$. Because only the source cells generate demand, the right-hand side of (3) is set as $d_{i}^{t-1}$ and the right-hand side of (4) is equal to 0 . Constraint (5) bounds the total outflow rate of a cell by its current occupancy. Constraint (6) represents that the total inflow rate of a cell is bounded by its remaining capacity. Constraints (7) and (8) ensure that the total inflow and outflow rate of a cell are limited by the expandable inflow and outflow capacity, respectively. The remaining constraints, from (9) to (13), report the initial conditions and nonnegativity conditions. Constraint (14) guarantees that the total cost of expansion is within the available budget.

\section{Affinely Adjustable Robust Formulation}

In this section, we firstly develop the adjustable robust counterpart (ARC) of the SODNDP model, which incorporates the demand uncertainty into a linear programming problem via the ARO approach. However, the formulation of ARC is intractable since it is a semi-infinite program. Then, we reformulate the ARC of SODNDP as a tractable linear programming by using the affine decision rules proposed by Ben-Tal et al. [22]. In the SODNDP, constraint (3) is the only set of constraints related to demand generation. Constraint (3) can be reformulated as the following inequality constraint $[1,36]$ :

$$
\begin{array}{r}
x_{i}^{t}-x_{i}^{t-1}-\sum_{k \in C} a_{k i} y_{k i}^{t-1}+\sum_{j \in C} a_{i j} y_{i j}^{t-1} \geq d_{i}^{t-1}, \\
\forall i \in C_{R}, t \in \mathfrak{\Im} .
\end{array}
$$

We can easily identify the optimal solution to the problem without constraint (15) which is zero for all $x$ and $y$. The introduction of constraint (15) makes zero no longer the optimal solution to the problem. Thus, constraint (15) is always binding at the optimal solution and it can replace constraint (3). Note that the vehicle holding phenomenon can also happen in this model since constraint (15) is always binding and two equations are equivalent in this model. Similarly, constraint (4) can be replaced by the following inequality constraint:

$$
\begin{array}{r}
x_{i}^{t}-x_{i}^{t-1}-\sum_{k \in C} a_{k i} y_{k i}^{t-1}+\sum_{j \in C} a_{i j} y_{i j}^{t-1} \geq 0, \\
\forall i \in C \backslash C_{R} \cup C_{S}, t \in \mathfrak{\Im} .
\end{array}
$$

Next, we assume that uncertain demand $d_{i}^{t-1}$ belongs to a prescribed uncertainty set. A box uncertainty set is used to bound demand uncertainty in Chung et al. [1]. However, BenTal et al. [34] pointed out that the box uncertainty set is overly conservative and adopted the polyhedral set as uncertain demand set. Similar to Ben-Tal et al. [34], we choose the following polyhedral set as the uncertainty set:

$$
\begin{aligned}
& d_{i}^{t} \in U=\left\{d_{i}^{t}:(1-\theta) \bar{d}_{i}^{t} \leq d_{i}^{t} \leq(1+\theta) \bar{d}_{i}^{t},\right. \\
&\left.\forall i \in C_{R}, t \in \mathfrak{I}, \sum_{t \in \mathfrak{I}} d_{i}^{t} \leq D_{i}, \forall i \in C_{R}\right\},
\end{aligned}
$$

where $\bar{d}_{i}^{t}$ is the nominal demand and $\theta$ is the demand uncertainty level. Next, we use the adjustable robust optimization methods to formulate the adjustable robust counterpart of SODNDP. Then, we have

SODNDP-ARC:

$$
\begin{aligned}
& \min _{b} \max _{d} \min _{x, y} \sum_{t \in \mathfrak{J}} \sum_{i \in C \backslash C_{s}} c_{i}^{t} x_{i}^{t}, \\
& \text { subject to } \\
& \begin{array}{c}
x_{i}^{t}-x_{i}^{t-1}-\sum_{k \in C} a_{k i} y_{k i}^{t-1} \\
+\sum_{j \in C} a_{i j} y_{i j}^{t-1} \geq d_{i}^{t-1},
\end{array} \\
& \forall i \in C_{R}, t \in \mathfrak{J}, \forall d_{i}^{t} \in U, \\
& x_{i}^{t}-x_{i}^{t-1}-\sum_{k \in C} a_{k i} y_{k i}^{t-1} \\
& +\sum_{j \in C} a_{i j} y_{i j}^{t-1} \geq 0 \\
& \forall i \in C \backslash C_{R} \cup C_{S}, t \in \mathfrak{J}, \\
& \sum_{j \in C} a_{i j} y_{i j}^{t} \leq x_{i}^{t} \\
& \forall i \in C \backslash C_{S}, t \in \mathfrak{J}, \\
& \sum_{k \in C} a_{k i} y_{k i}^{t} \leq \delta_{i}^{t}\left(N_{i}^{t}+\chi_{i} b_{i}-x_{i}^{t}\right) \\
& \forall i \in C \backslash C_{R} \cup C_{S}, t \in \mathfrak{J}, \\
& \sum_{k \in C} a_{k i} y_{k i}^{t} \leq Q_{i}^{t}+\phi_{i} b_{i} \\
& \forall i \in C \backslash C_{R} \cup C_{S}, t \in \mathfrak{J}, \\
& \sum_{j \in C} a_{i j} y_{i j}^{t} \leq Q_{i}^{t}+\phi_{i} b_{i} \\
& \forall i \in C \backslash C_{S}, t \in \mathfrak{J}, \\
& x_{i}^{0}=\widehat{x}_{i} \quad \forall i \in C \backslash C_{S}, \\
& y_{i j}^{0}=0, \quad \forall(i, j) \in C \times C, \\
& x_{i}^{t} \geq 0, \quad \forall i \in C \backslash C_{S}, t \in \mathfrak{\Im},
\end{aligned}
$$




$$
\begin{aligned}
& y_{i j}^{t} \geq 0, \quad \forall(i, j) \in C \times C, t \in \mathfrak{J}, \\
& b_{i} \geq 0, \quad \forall i \in C \backslash C_{S}, \\
& \sum_{i \in C \backslash C_{s}} b_{i} \leq B .
\end{aligned}
$$

Ben-Tal et al. [22] showed that the ARC is more flexible than the RC; however, in most cases the ARC is computationally intractable (NP-hard). To address this difficulty, Ben-Tal et al. [22] proposed the affine decision rules; that is, adjustable variables are restricted to be affine function of the uncertain data. Here, we use the affine decision rules proposed by BenTal et al. [22] to make ARC computationally tractable. Then,

$$
\begin{aligned}
& x_{i}^{t}=\eta_{i t}^{-1}+\sum_{s \in C_{R}} \sum_{\tau \in I_{t}} \eta_{i t}^{s \tau} d_{s}^{\tau}, \\
& y_{i j}^{t}=\pi_{i j t}^{-1}+\sum_{s \in C_{R}} \sum_{\tau \in I_{t}} \pi_{i j t}^{s \tau} d_{s}^{\tau},
\end{aligned}
$$

where $y_{i j}^{t}$ is adjustable control variable, $x_{i}^{t}$ is state variable, $\eta_{i t}^{-1}, \pi_{i j t}^{-1}, \eta_{i t}^{s \tau}$, and $\pi_{i j t}^{s \tau}$ are nonadjustable variables, and $I_{t}=$ $\{0,1,2, \ldots, t-1\}$. By substituting the state and control variable, we have the following SODNP-AARC formulation:

$$
\text { SODNP-AARC: }
$$

$$
\begin{aligned}
& \min _{\eta, \pi, b, z} z \\
& \text { s.t. } \quad \sum_{t \in \mathfrak{\Im}} \sum_{i \in C \backslash C_{s}} c_{i}^{t}\left(\eta_{i t}^{-1}+\sum_{s \in C_{R}} \sum_{\tau \in I_{t}} \eta_{i t}^{s \tau} d_{s}^{\tau}\right) \leq z, \quad \forall d_{s}^{\tau} \in U, \\
& \left(\eta_{i t}^{-1}+\sum_{s \in C_{R}} \sum_{\tau \in I_{t}} \eta_{i t}^{s \tau} d_{s}^{\tau}\right) \\
& -\left(\eta_{i t-1}^{-1}+\sum_{s \in C_{R}} \sum_{\tau \in I_{t-1}} \eta_{i t-1}^{s \tau} d_{s}^{\tau}\right) \\
& -\sum_{k \in C} a_{k i}\left(\pi_{k i t-1}^{-1}+\sum_{s \in C_{R}} \sum_{t \in I_{t-1}} \pi_{k i t-1}^{s \tau} d_{s}^{\tau}\right) \\
& +\sum_{j \in C} a_{i j}\left(\pi_{i j t}^{-1}+\sum_{s \in C_{R}} \sum_{t \in I_{t}} \pi_{i j t}^{s \tau} d_{s}^{\tau}\right) \geq d_{i}^{t-1}, \\
& \forall i \in C_{R}, t \in \mathfrak{J}, d_{i}^{t} \in U, \\
& \left(\eta_{i t}^{-1}+\sum_{s \in C_{R}} \sum_{\tau \in I_{t}} \eta_{i t}^{s \tau} d_{s}^{\tau}\right) \\
& -\left(\eta_{i t-1}^{-1}+\sum_{s \in C_{R}} \sum_{\tau \in I_{t-1}} \eta_{i t-1}^{s \tau} d_{s}^{\tau}\right) \\
& -\sum_{k \in C} a_{k i}\left(\pi_{k i t-1}^{-1}+\sum_{s \in C_{R}} \sum_{t \in I_{t-1}} \pi_{k i t-1}^{s \tau} d_{s}^{\tau}\right)
\end{aligned}
$$

$$
\begin{array}{r}
+\sum_{j \in C} a_{i j}\left(\pi_{i j t}^{-1}+\sum_{s \in C_{R}} \sum_{t \in I_{t}} \pi_{i j t}^{s \tau} d_{s}^{\tau}\right) \geq 0, \\
\forall i \in C \backslash C_{R} \cup C_{S}, t \in \mathfrak{J}, d_{i}^{t} \in U, \\
\sum_{j \in C} a_{i j}\left(\pi_{i j t}^{-1}+\sum_{s \in C_{R}} \sum_{\tau \in I_{t}} \pi_{i j t}^{s \tau} d_{s}^{\tau}\right) \\
\leq\left(\eta_{i t}^{-1}+\sum_{s \in C_{R}} \sum_{\tau \in I_{t}} \eta_{i t}^{s \tau} d_{s}^{\tau}\right),
\end{array}
$$$$
\forall i \in C \backslash C_{S}, t \in \mathfrak{\Im}, d_{i}^{t} \in U \text {, }
$$$$
\begin{aligned}
& \sum_{k \in C} a_{k i}\left(\pi_{k i t}^{-1}+\sum_{s \in C_{R}} \sum_{\tau \in I_{t}} \pi_{k i t}^{s \tau} d_{s}^{\tau}\right) \\
& \quad \leq \delta_{i}^{t}\left(N_{i}^{t}+\chi_{i} b_{i}-\left(\eta_{i t}^{-1}+\sum_{s \in C_{R}} \sum_{\tau \in I_{t}} \eta_{i t}^{s \tau} d_{s}^{\tau}\right)\right),
\end{aligned}
$$$$
\forall i \in C \backslash C_{R} \cup C_{S}, t \in \mathfrak{I}, d_{i}^{t} \in U,
$$$$
\sum_{k \in C} a_{k i}\left(\pi_{k i t}^{-1}+\sum_{s \in C_{R}} \sum_{\tau \in I_{t}} \pi_{k i t}^{s \tau} d_{s}^{\tau}\right) \leq Q_{i}^{t}+\phi_{i} b_{i},
$$$$
\forall i \in C \backslash C_{R} \cup C_{S}, t \in \mathfrak{I}, d_{i}^{t} \in U,
$$$$
\sum_{k \in C} a_{k i}\left(\pi_{k i t}^{-1}+\sum_{s \in C_{R}} \sum_{\tau \in I_{t}} \pi_{k i t}^{s \tau} d_{s}^{\tau}\right)
$$$$
\leq Q_{i}^{t}+\phi_{i} b_{i}, \quad \forall i \in C \backslash C_{S}, t \in \mathfrak{J}, d_{i}^{t} \in U
$$$$
\eta_{i 0}^{-1}=\widehat{x}_{i}, \quad \forall i \in C \backslash C_{S},
$$$$
\pi_{i j 0}^{-1}=0, \quad \forall(i, j) \in C \times C,
$$$$
\eta_{i t}^{-1}+\sum_{s \in C_{R}} \sum_{\tau \in I_{t}} \eta_{i t}^{s \tau} d_{s}^{\tau} \geq 0
$$$$
\forall i \in C \backslash C_{S}, t \in \mathfrak{I}, \tilde{d}_{i}^{t} \in U \text {, }
$$$$
\pi_{i j t}^{-1}+\sum_{s \in C_{R}} \sum_{t \in I_{t}} \pi_{i j t}^{s \tau} d_{s}^{\tau} \geq 0
$$$$
\forall(i, j) \in C \times C, t \in \mathfrak{\Im}, \tilde{d}_{i}^{t} \in U,
$$$$
\sum_{i \in C \backslash C_{s}} b_{i} \leq B
$$$$
b_{i} \geq 0, \quad \forall i \in C \backslash C_{S} .
$$

It can be seen from the above formulation that SODNPAARC is intractable since it is a semi-infinite program, and it can be reformulated as a tractable optimization problem as shown in Theorem 1. 
Theorem 1. Given a polyhedral uncertainty set $U$, the affinely adjustable robust counterpart of the SODNDP becomes the following linear programming and thus computationally tractable. Note that $\lambda$ is a set of dual variables and the numerical indexes are used for notational simplicity:

SODNDP-AARC1:

$$
\min _{b, z, \lambda, \pi, \eta} z
$$

s.t. $\quad \sum_{t \in \mathfrak{\Im}} \sum_{i \in C \backslash C_{s}} c_{i}^{t} \eta_{i t}^{-1}-\sum_{s \in C_{R}} \sum_{\tau \in \mathfrak{J}}(1-\theta) \lambda_{s \tau}^{11} \bar{d}_{s}^{\tau}$

$$
\begin{gathered}
+\sum_{s \in C_{R}} \sum_{\tau \in \Im}(1+\theta) \lambda_{s \tau}^{12} \bar{d}_{s}^{\tau}+\sum_{s \in C_{R}} \lambda_{s}^{13} D_{s} \leq z, \\
\lambda_{s \tau}^{12}-\lambda_{s \tau}^{11}+\lambda_{s}^{13}=\sum_{i \in C \backslash C_{s}} \sum_{t=\{\tau+1, \ldots, T\}} c_{i}^{t} \eta_{i t}^{s \tau},
\end{gathered}
$$$$
\forall s \in C_{R}, \tau \in\{0,1,2, \ldots, T-1\},
$$$$
\lambda_{s \tau}^{12}-\lambda_{s \tau}^{11}+\lambda_{s}^{13}=0, \quad \forall s \in C_{R}, \tau \in\{T\},
$$$$
\lambda_{s \tau}^{12}, \lambda_{s \tau}^{11}, \lambda_{s}^{13} \geq 0, \quad \forall s \in C_{R}, \tau \in \mathfrak{I},
$$$$
\eta_{i t}^{-1}-\eta_{i t-1}^{-1}-\sum_{k \in C} a_{k i} \pi_{k i t-1}^{-1}+\sum_{j \in C} a_{i j} \pi_{i j t-1}^{-1}
$$$$
+\sum_{s \in C_{R}, \tau \in \mathfrak{I}} \lambda_{i t s \tau}^{21}(1-\theta) \bar{d}_{s}^{\tau}
$$$$
-\sum_{s \in C_{R}, \tau \in \mathfrak{J}} \lambda_{i t s \tau}^{22}(1+\theta) \bar{d}_{s}^{\tau}
$$$$
-\sum_{s \in C_{R}} \lambda_{i t s}^{23} D_{s} \geq 0, \quad \forall i \in C_{R}, t \in \mathfrak{J},
$$$$
\lambda_{i t s \tau}^{22}-\lambda_{i t s \tau}^{21}+\lambda_{i t s}^{23}=I_{\{\tau=t-1, s=i\}}-\eta_{i t}^{s \tau}
$$$$
+\left(\eta_{i t-1}^{s \tau}+\sum_{k \in C} a_{k i} \pi_{k i t-1}^{s \tau}-\sum_{j \in C} a_{i j} \pi_{i j t-1}^{s \tau}\right)
$$$$
\times I_{\{\tau<t-1\}}, \quad \forall i \in C_{R}, t \in \mathfrak{I},
$$$$
s \in C_{R}, \tau \in\{0,1, \ldots, t-1\},
$$$$
\lambda_{i t s \tau}^{22}-\lambda_{i t s \tau}^{21}+\lambda_{i t s}^{23}=0 \text {, }
$$

$\forall i \in C_{R}, t \in \mathfrak{J}, s \in C_{R}, \tau \in\{t, \ldots, T\}$,

$$
\lambda_{i t s \tau}^{21}, \lambda_{i t s \tau}^{22}, \lambda_{i t s}^{23} \geq 0 \text {, }
$$

$\forall i \in C_{R}, t \in \mathfrak{J}, s \in C_{R}, \tau \in \mathfrak{J}$,

$$
\begin{aligned}
\eta_{i t}^{-1} & -\eta_{i t-1}^{-1}-\sum_{k \in C} a_{k i} \pi_{k i t-1}^{-1}+\sum_{j \in C} a_{i j} \pi_{i j t-1}^{-1} \\
& +\sum_{s \in C_{R}, \tau \in \mathfrak{I}} \lambda_{i t s \tau}^{31}(1-\theta) \bar{d}_{s}^{\tau}-\sum_{s \in C_{R}, \tau \in \mathfrak{I}} \lambda_{i t s \tau}^{32}(1+\theta) \bar{d}_{s}^{\tau} \\
& -\sum_{s \in C_{R}} \lambda_{i t s}^{33} D_{s} \geq 0, \quad \forall i \in C \backslash C_{R} \cup C_{S}, \quad t \in \mathfrak{J},
\end{aligned}
$$

$$
\begin{gathered}
\lambda_{i t s \tau}^{32}-\lambda_{i t s \tau}^{31}+\lambda_{i t s}^{33}=-\eta_{i t}^{s \tau} \\
+\left(\eta_{i t-1}^{s \tau}+\sum_{k \in C} a_{k i} \pi_{k i t-1}^{s \tau}-\sum_{j \in C} a_{i j} \pi_{i j t-1}^{s \tau}\right) \\
\times I_{\{\tau<t-1\}}, \quad \forall i \in C \backslash C_{R} \cup C_{S}, \quad t \in \mathfrak{J}, \\
s \in C_{R}, \quad \tau \in\{0,1, \ldots, t-1\}, \\
\lambda_{i t s \tau}^{32}-\lambda_{i t s \tau}^{31}+\lambda_{i t s}^{33}=0, \quad \forall i \in C \backslash C_{R} \cup C_{S}, \\
t \in \mathfrak{J}, \quad s \in C_{R}, \tau \in\{t, \ldots, T\}, \\
\lambda_{i t s \tau}^{31}, \lambda_{i t s \tau}^{32}, \lambda_{i t s}^{33} \geq 0, \quad \forall i \in C \backslash C_{R} \cup C_{S}, \\
t \in \mathfrak{J}, \quad s \in C_{R}, \tau \in \mathfrak{J},
\end{gathered}
$$

$$
\begin{gathered}
\sum_{j \in C} a_{i j} \pi_{i j t}^{-1}-\eta_{i t}^{-1}+\sum_{s \in C_{R}, \tau \in \mathfrak{I}} \lambda_{i t s \tau}^{42}(1+\theta) \bar{d}_{s}^{\tau} \\
-\sum_{s \in C_{R}, \tau \in \mathfrak{I}} \lambda_{i t s \tau}^{41}(1-\theta) \bar{d}_{s}^{\tau}+\sum_{s \in C_{R}} \lambda_{i t s}^{43} D_{s} \leq 0,
\end{gathered}
$$

$\forall i \in C \backslash C_{S}, t \in \mathfrak{J}$,

$\lambda_{i t s \tau}^{42}-\lambda_{i t s \tau}^{41}+\lambda_{i t s}^{43}=\sum_{j \in C} a_{i j} \pi_{i j t}^{s \tau}-\eta_{i t}^{s \tau}$,

$\forall i \in C \backslash C_{S}, t \in \mathfrak{J}, s \in C_{R}, \tau \in\{0,1, \ldots, t-1\}$,

$\lambda_{i t s \tau}^{42}-\lambda_{i t s \tau}^{41}+\lambda_{i t s}^{43}=0, \quad \forall i \in C \backslash C_{S}$,

$t \in \mathfrak{J}, s \in C_{R}, \tau \in\{t, \ldots, T\}$,

$\lambda_{i t s \tau}^{41}, \lambda_{i t s \tau}^{42}, \lambda_{i t s}^{43} \geq 0, \quad \forall i \in C \backslash C_{S}$,

$$
t \in \mathfrak{I}, s \in C_{R}, \tau \in \mathfrak{J},
$$

$$
\begin{aligned}
& \sum_{k \in C} a_{k i} \pi_{k i t}^{-1}+\sum_{s \in C_{R}, \tau \in \mathfrak{I}} \lambda_{i t s \tau}^{52}(1+\theta) \bar{d}_{s}^{\tau} \\
& \quad-\sum_{s \in C_{R}, \tau \in \mathfrak{I}} \lambda_{i t s \tau}^{51}(1-\theta) \bar{d}_{s}^{\tau} \\
& \quad+\sum_{s \in C_{R}} \lambda_{i t s}^{53} D_{s} \leq \delta_{i}^{t}\left(N_{i}^{t}+\chi_{i} b_{i}-\eta_{i t}^{-1}\right),
\end{aligned}
$$

$\forall i \in C \backslash C_{R} \cup C_{S}, t \in \mathfrak{I}$

$$
\lambda_{i t s \tau}^{52}-\lambda_{i t s \tau}^{51}+\lambda_{i t s}^{53}=\sum_{k \in C} a_{k i} \pi_{k i t}^{s \tau}+\delta_{i}^{t} \eta_{i t}^{s \tau},
$$

$$
\forall i \in C \backslash C_{R} \cup C_{S}, t \in \mathfrak{J},
$$

$$
s \in C_{R}, \tau \in\{0,1, \ldots, t-1\},
$$

$\lambda_{i t s \tau}^{52}-\lambda_{i t s \tau}^{51}+\lambda_{i t s}^{53}=0, \quad \forall i \in C \backslash C_{R} \cup C_{S}$,

$$
t \in \mathfrak{I}, s \in C_{R}, \tau \in\{t, \ldots, T\},
$$

$\lambda_{i t s \tau}^{51}, \lambda_{i t s \tau}^{52}, \lambda_{i t s}^{53} \geq 0, \quad \forall i \in C \backslash C_{R} \cup C_{S}$,

$$
t \in \mathfrak{J}, s \in C_{R}, \tau \in \mathfrak{I},
$$




$$
\begin{gathered}
\sum_{k \in C} a_{k i} \pi_{k i t}^{-1}+\sum_{s \in C_{R}, \tau \in \mathfrak{I}} \lambda_{i t s \tau}^{62}(1+\theta) \bar{d}_{s}^{\tau} \\
-\sum_{s \in C_{R}, \tau \in \mathfrak{I}} \lambda_{i t s \tau}^{61}(1-\theta) \bar{d}_{s}^{\tau} \\
+\sum_{s \in C_{R}} \lambda_{i t s}^{63} D_{s} \leq Q_{i}+\phi_{i} b_{i},
\end{gathered}
$$

$\forall i \in C \backslash C_{R} \cup C_{S}, t \in \mathfrak{J}$,

$$
\lambda_{i t s \tau}^{62}-\lambda_{i t s \tau}^{61}+\lambda_{i t s}^{63}=\sum_{k \in C} a_{k i} \pi_{k i t}^{s \tau},
$$$$
\forall i \in C \backslash C_{R} \cup C_{S}, t \in \mathfrak{I},
$$

$s \in C_{R}, \tau \in\{0,1, \ldots, t-1\}$,

$\lambda_{i t s \tau}^{62}-\lambda_{i t s \tau}^{61}+\lambda_{i t s}^{63}=0, \quad \forall i \in C \backslash C_{R} \cup C_{S}$,

$$
t \in \mathfrak{J}, s \in C_{R}, \tau \in\{t, \ldots, T\},
$$

$\lambda_{i t s \tau}^{61}, \lambda_{i t s \tau}^{62}, \lambda_{i t s}^{63} \geq 0, \quad \forall i \in C \backslash C_{R} \cup C_{S}$,

$$
t \in \mathfrak{I}, s \in C_{R}, \tau \in \mathfrak{J},
$$

$$
\begin{aligned}
& \sum_{j \in C} a_{i j} \pi_{i j t}^{-1}+\sum_{s \in C_{R}, \tau \in \mathfrak{I}} \lambda_{i t s \tau}^{72}(1+\theta) \bar{d}_{s}^{\tau} \\
& -\sum_{s \in C_{R}, \tau \in \mathfrak{I}} \lambda_{i t s \tau}^{71}(1-\theta) \bar{d}_{s}^{\tau} \\
& +\sum_{s \in C_{R}} \lambda_{i t s}^{73} D_{s} \leq Q_{i}+\phi_{i} b_{i}, \\
& \forall i \in C \backslash C_{S}, \quad t \in \mathfrak{I}, \\
& \lambda_{i t s \tau}^{72}-\lambda_{i t s \tau}^{71}+\lambda_{i t s}^{73}=\sum_{j \in C} a_{i j} \pi_{i j t}^{s \tau}, \\
& \quad \forall i \in C \backslash C_{S}, \quad t \in \mathfrak{J}, \\
& s \in C_{R}, \quad \tau \in\{0,1, \ldots, t-1\}, \\
& \lambda_{i t s \tau}^{72}-\lambda_{i t s \tau}^{71}+\lambda_{i t s}^{73}=0, \quad \forall i \in C \backslash C_{S}, t \in \mathfrak{J}, \\
& \quad s \in C_{R}, \quad \tau \in\{t, \ldots, T\}, \\
& \lambda_{i t s \tau}^{71}, \lambda_{i t s \tau}^{72}, \lambda_{i t s}^{73} \geq 0,
\end{aligned}
$$$$
\forall i \in C \backslash C_{S}, t \in \mathfrak{I}, s \in C_{R}, \tau \in \mathfrak{I},
$$$$
\eta_{i t}^{-1}-\sum_{s \in C_{R}, \tau \in \mathfrak{I}} \lambda_{i t s \tau}^{82}(1+\theta) \bar{d}_{s}^{\tau}
$$$$
+\sum_{s \in C_{R}, \tau \in \mathfrak{I}} \lambda_{i t s \tau}^{81}(1-\theta) \bar{d}_{s}^{\tau}
$$$$
-\sum_{s \in C_{R}} \lambda_{i t s}^{83} D_{s} \geq 0, \quad \forall i \in C \backslash C_{S}, t \in \mathfrak{I},
$$$$
\lambda_{i t s \tau}^{82}-\lambda_{i t s \tau}^{81}+\lambda_{i t s}^{83}=-\eta_{i t}^{s \tau}, \quad \forall i \in C \backslash C_{S},
$$$$
t \in \mathfrak{J}, s \in C_{R}, \tau \in\{0,1, \ldots, t-1\},
$$

$$
\begin{aligned}
& \lambda_{i t s \tau}^{82}-\lambda_{i t s \tau}^{81}+\lambda_{i t s}^{83}=0, \quad \forall i \in C \backslash C_{S}, \\
& t \in \mathfrak{I}, \quad s \in C_{R}, \quad \tau \in\{t, \ldots, T\}, \\
& \lambda_{i t s \tau}^{81}, \lambda_{i t s \tau}^{82}, \lambda_{i t s}^{83} \geq 0, \\
& \forall i \in C \backslash C_{S}, \quad t \in \mathfrak{I}, \quad s \in C_{R}, \tau \in \mathfrak{I}, \\
& \pi_{i j t}^{-1}-\sum_{s \in C_{R}, \tau \in \mathfrak{I}} \lambda_{i j t s \tau}^{92}(1+\theta) \bar{d}_{s}^{\tau} \\
& +\sum_{s \in C_{R}, \tau \in \mathfrak{I}} \lambda_{i j t s \tau}^{91}(1-\theta) \bar{d}_{s}^{\tau}-\sum_{s \in C_{R}} \lambda_{i t s}^{93} D_{s} \geq 0, \\
& \quad \forall(i, j) \in\{a(i, j)=1\}, t \in \mathfrak{I},
\end{aligned}
$$

$$
\begin{array}{r}
\lambda_{i j t s \tau}^{92}-\lambda_{i j t s \tau}^{91}+\lambda_{i j t s}^{93}=-\pi_{i j t}^{s \tau}, \quad \forall(i, j) \in\{a(i, j)=1\}, \\
t \in \mathfrak{J}, s \in C_{R}, \tau \in\{0,1, \ldots, t-1\},
\end{array}
$$

$$
\begin{array}{r}
\lambda_{i j t s \tau}^{92}-\lambda_{i j t s \tau}^{91}+\lambda_{i j t s}^{93}=0, \quad \forall(i, j) \in\{a(i, j)=1\}, \\
t \in \mathfrak{J}, \quad s \in C_{R}, \tau \in\{t, \ldots, T\},
\end{array}
$$

$$
\begin{array}{r}
\lambda_{i j t s \tau}^{91}, \lambda_{i j t s \tau}^{92}, \lambda_{i j t s}^{93} \geq 0, \quad \forall(i, j) \in\{a(i, j)=1\}, \\
t \in \mathfrak{J}, s \in C_{R}, \tau \in \mathfrak{J},
\end{array}
$$

$\eta_{i 0}^{-1}=\widehat{x}_{i}, \quad \forall i \in C \backslash C_{S}$,

$\pi_{i j 0}^{-1}=0, \quad \forall(i, j) \in\{a(i, j)=1\}$,

$\sum_{i \in C \backslash C_{s}} b_{i} \leq B$

$b_{i} \geq 0, \quad \forall i \in C \backslash C_{S}$,

where $I_{\{\tau=t-1, s=i\}}$ in the constraint (28) denotes that if $\tau=t-$ $1, s=i$, then $I_{\{\tau=t-1, s=i\}}=1$; otherwise $I_{\{\tau=t-1, s=i\}}=0$, and $I_{\{\tau<t-1\}}$ in the constraint (28) denotes that if $\tau<t-1$, then $I_{\{\tau<t-1\}}=1$; otherwise $I_{\{\tau<t-1\}}=0$.

Proof. By applying the following relationship, we can reformulate each constraint affected by demand uncertainty as an equivalent LP problem. For example,

$$
\begin{aligned}
& \sum_{t \in \mathfrak{\Im}} \sum_{i \in C \mid C_{s}} c_{i}^{t}\left(\eta_{i t}^{-1}+\sum_{s \in C_{R}} \sum_{\tau \in I_{t}} \eta_{i t}^{s \tau} d_{s}^{\tau}\right) \leq z, \\
& \forall d_{s}^{\tau} \in U=\left\{(1-\theta) \bar{d}_{s}^{\tau} \leq d_{s}^{\tau} \leq(1+\theta) \bar{d}_{s}^{\tau}, \forall s \in C_{R}, \tau \in \mathfrak{J},\right.
\end{aligned}
$$$$
\left.\sum_{\tau \in \mathfrak{T}} d_{s}^{\tau} \leq D_{s}, \forall s \in C_{R}\right\}
$$$$
\Longleftrightarrow \max _{d_{s}^{\tau} \in U_{t \in \Im}} \sum_{i \in C \mid C_{s}} c_{i}^{t}\left(\eta_{i t}^{-1}+\sum_{s \in C_{R}} \sum_{\tau \in I_{t}} \eta_{i t}^{s \tau} d_{s}^{\tau}\right) \leq z .
$$ 
According to strong duality property, we can derive the equivalent constraint with dual problem of the above problem:

$$
\begin{aligned}
& \max _{d_{s}^{\tau}} \sum_{t \in \Im} \sum_{i \in C \backslash C_{s}} c_{i}^{t}\left(\eta_{i t}^{-1}+\sum_{s \in C_{R}} \sum_{\tau \in I_{t}} \eta_{i t}^{s \tau} d_{s}^{\tau}\right) \leq z \\
& \text { s.t. } \quad(1-\theta) \bar{d}_{s}^{\tau} \leq d_{s}^{\tau} \leq(1+\theta) \bar{d}_{s}^{\tau}, \quad \forall s \in C_{R}, \quad \tau \in \mathfrak{J} \\
& \sum_{\tau \in \mathfrak{I}} d_{s}^{\tau} \leq D_{s}, \quad \forall s \in C_{R} \\
& \mathbb{1} \\
& \min _{\eta_{i t}^{-1}, \lambda_{s \tau}^{11}, \lambda_{s \tau}^{12}, \lambda_{s}^{13}} \sum_{i \in C \backslash C_{S}, t \in \mathfrak{I}} c_{i}^{t} \eta_{i t}^{-1}-\sum_{s \in C_{R}, \tau \in \mathfrak{I}}(1-\theta) \lambda_{s \tau}^{11} \bar{d}_{s}^{\tau} \\
& +\sum_{s \in C_{R}, \tau \in \mathfrak{I}}(1+\theta) \lambda_{s \tau}^{12} \bar{d}_{s}^{\tau}+\sum_{s \in C_{R}} \lambda_{s}^{13} D_{s} \leq z \\
& \text { s.t. } \quad \lambda_{s \tau}^{12}-\lambda_{s \tau}^{11}+\lambda_{s}^{13}=\sum_{i \in C \backslash C_{s}} \sum_{t=\{\tau+1, \ldots, T\}} c_{i}^{t} \eta_{i t}^{s \tau} \text {, } \\
& \forall s \in C_{R}, \tau \in\{0,1,2, \ldots, T-1\}, \\
& \lambda_{s \tau}^{12}-\lambda_{s \tau}^{11}+\lambda_{s}^{13}=0, \quad \forall s \in C_{R}, \quad \tau \in\{T\} \\
& \lambda_{s \tau}^{12}, \lambda_{s \tau}^{11}, \lambda_{s}^{13} \geq 0, \quad \forall s \in C_{R}, \tau \in \Im
\end{aligned}
$$

where $\lambda_{s \tau}^{11}, \lambda_{s \tau}^{12}$, and $\lambda_{s}^{13}$ are dual variables. Therefore, we can derive the constraints (23)-(26). Similarity, the other semiinfinite constraints can also write as the equivalent linear constraints.

Note that the optimal objective of SODNDP-AARC1 is a guaranteed upper bound value for all realization of uncertain data under the assumption of linear dependency. It also can be interpreted as the optimistic estimate of total cost in worst case, which can be lower than the optimistic estimate from robust counterpart as SODNDP-AARC1 has a larger robust feasible region [22]. Moreover, the decision variables of SODNDP-AARC1 are not adjustable control and state variables but a set of coefficient of affine function of the control variables including $\pi_{i j t}^{-1}, \pi_{i j t}^{s \tau}, \eta_{i t}^{-1}$, and $\eta_{i t}^{s \tau}$. It implies that the solution of SODNDP-AARC1 is a linear decision rule. Specific values of $x_{i}^{t}$ and $y_{i j}^{t}$ are computed after the realization of the demand at time $t-1$ [34].

\section{Robust Formulation}

To demonstrate that the AARC is more flexible, we use the usual RO approach to develop the RC of SODNDP in this section. We note that constraint (11) is redundant since constraints (5) and (12) guarantee the nonnegativity of $x_{i}^{t}$. By using constraints (3), (4), (9), and (10), $x_{i}^{t}$ can be represented as follows:

$$
x_{i}^{t}=\widehat{x}_{i}+\sum_{\tau=0}^{t-1}\left(\sum_{k \in C} a_{k i} y_{k i}^{\tau}-\sum_{j \in C} a_{i j} y_{i j}^{\tau}+d_{i}^{\tau}\right),
$$

$\forall i \in C_{R}, t \in \mathfrak{I}$

$$
x_{i}^{t}=\widehat{x}_{i}+\sum_{\tau=0}^{t-1}\left(\sum_{k \in C} a_{k i} y_{k i}^{\tau}-\sum_{j \in C} a_{i j} y_{i j}^{\tau}\right)
$$

$\forall i \in C \backslash C_{R} \cup C_{S}, t \in \mathfrak{\Im}$.

As before, it is also assumed that the demand uncertainty belongs to the uncertain set $U$. Thus, we can get the following RC of SODNDP: SODNDP-RC:

$$
\begin{array}{ll}
\min _{b, y, z} & z, \\
\text { s.t. } & \sum_{t \in \Im} \sum_{i \in C_{R}} c_{i}^{t}
\end{array}
$$

$$
\begin{aligned}
& \times\left(\widehat{x}_{i}+\sum_{\tau=0}^{t-1}\left(\sum_{k \in C} a_{k i} y_{k i}^{\tau}-\sum_{j \in C} a_{i j} y_{i j}^{\tau}+d_{i}^{\tau}\right)\right) \\
& +\sum_{t \in \Im} \sum_{i \in C \backslash C_{R} \cup C_{S}} c_{i}^{t}
\end{aligned}
$$$$
\times\left(\widehat{x}_{i}+\sum_{\tau=0}^{t-1}\left(\sum_{k \in C} a_{k i} y_{k i}^{\tau}-\sum_{j \in C} a_{i j} y_{i j}^{\tau}\right)\right)
$$$$
\leq z, \quad \forall d_{i}^{t} \in U,
$$$$
\sum_{j \in C} a_{i j} y_{i j}^{t}
$$

$$
\begin{array}{r}
\leq \widehat{x}_{i}+\sum_{\tau=0}^{t-1}\left(\sum_{k \in C} a_{k i} y_{k i}^{\tau}-\sum_{j \in C} a_{i j} y_{i j}^{\tau}+d_{i}^{\tau}\right), \\
\forall i \in C_{R}, t \in \mathfrak{\Im}, d_{i}^{t} \in U,
\end{array}
$$

$\sum_{j \in C} a_{i j} y_{i j}^{t}$

$$
\begin{array}{r}
\leq \widehat{x}_{i}+\sum_{\tau=0}^{t-1}\left(\sum_{k \in C} a_{k i} y_{k i}^{\tau}-\sum_{j \in C} a_{i j} y_{i j}^{\tau}\right), \\
\forall i \in C \backslash C_{R} \cup C_{S}, t \in \mathfrak{I}
\end{array}
$$




$$
\begin{aligned}
& \sum_{k \in C} a_{k i} y_{k i}^{t} \\
& \leq \delta_{i}^{t}\left(N_{i}^{t}+\chi_{i} b_{i}-\widehat{x}_{i}\right. \\
& \left.-\sum_{\tau=0}^{t-1}\left(\sum_{k \in C} a_{k i} y_{k i}^{\tau}-\sum_{j \in C} a_{i j} y_{i j}^{\tau}\right)\right), \\
& \forall i \in C \backslash C_{R} \cup C_{S}, t \in \mathfrak{\Im} \text {, } \\
& \sum_{k \in C} a_{k i} y_{k i}^{t} \leq Q_{i}^{t}+\phi_{i} b_{i}, \quad \forall i \in C \backslash C_{R} \cup C_{S}, t \in \mathfrak{J}, \\
& \sum_{j \in C} a_{i j} y_{i j}^{t} \leq Q_{i}^{t}+\phi_{i} b_{i}, \quad \forall i \in C \backslash C_{S}, t \in \mathfrak{I} \\
& y_{i j}^{0}=0, \quad \forall(i, j) \in C \times C, \\
& y_{i j}^{t} \geq 0, \quad \forall(i, j) \in C \times C, t \in \mathfrak{J}, \\
& \sum_{i \in C \backslash C_{s}} b_{i} \leq B \\
& b_{i} \geq 0, \quad \forall i \in C \backslash C_{S} .
\end{aligned}
$$

It is obvious that $\mathrm{RC}$ is also a semi-infinite problem and has infinitely many constraints. Similar to Theorem 1, we can convert it into the following tractable equivalent deterministic problem:

SODNDP-RC1:

$\min _{b, y, \mu, z} z$

$$
\begin{aligned}
& \text { s.t. } \quad \sum_{t \in \mathfrak{I}} \sum_{i \in C \backslash C_{S}} c_{i}^{t} \\
& \quad \times\left(\widehat{x}_{i}+\sum_{\tau=0}^{t-1}\left(\sum_{k \in C} a_{k i} y_{k i}^{\tau}-\sum_{j \in C} a_{i j} y_{i j}^{\tau}\right)\right) \\
& \quad-\sum_{s \in C_{R}, \tau \in \mathfrak{I}} \mu_{s \tau}^{11}(1-\theta) \bar{d}_{s}^{\tau}+\sum_{s \in C_{R}, \tau \in \mathfrak{I}} \mu_{s \tau}^{12}(1+\theta) \bar{d}_{s}^{\tau} \\
& \quad+\sum_{s \in C_{R}} \mu_{s}^{13} D_{s} \leq z, \\
& \mu_{s \tau}^{12}-\mu_{s \tau}^{11}+\mu_{s}^{13}=\sum_{t=\{\tau+1, \ldots, T\}} c_{s}^{t}, \\
& \forall s \in C_{R}, \tau \in\{0,1,2, \ldots, T-1\}, \\
& \mu_{s \tau}^{12}-\mu_{s \tau}^{11}+\mu_{s}^{13}=0, \quad \forall s \in C_{R}, \tau \in\{T\}, \\
& \mu_{s \tau}^{11}, \mu_{s \tau}^{12}, \mu_{s}^{13} \geq 0, \quad \forall s \in C_{R}, \tau \in \mathfrak{J},
\end{aligned}
$$

$$
\begin{aligned}
\sum_{j \in C} a_{i j} y_{i j}^{t} & \\
\leq & \widehat{x}_{i}+\sum_{\tau=0}^{t-1}\left(\sum_{k \in C} a_{k i} y_{k i}^{\tau}-\sum_{j \in C} a_{i j} y_{i j}^{\tau}\right) \\
& +\sum_{s \in C_{R}} \sum_{\tau \in \mathfrak{I}} \mu_{i t s \tau}^{21}(1-\theta) \bar{d}_{s}^{\tau} \\
& -\sum_{s \in C_{R}} \sum_{\tau \in \mathfrak{J}} \mu_{i t s \tau}^{22}(1+\theta) \bar{d}_{s}^{\tau} \\
& -\sum_{s \in C_{R}} \mu_{i t s}^{23} D_{s}, \quad \forall i \in C_{R}, t \in \mathfrak{J},
\end{aligned}
$$

$$
\begin{aligned}
& \mu_{i t s \tau}^{22}-\mu_{i t s \tau}^{21}+\mu_{i t s}^{23}+I_{\{i=s, \tau<t\}}=0, \\
& \forall i \in C_{R}, t \in \mathfrak{J}, s \in C_{R}, \tau \in \mathfrak{J}, \\
& \mu_{i t s \tau}^{21}, \mu_{i t s \tau}^{22}, \mu_{i t s}^{23} \geq 0, \quad \forall i \in C_{R}, \quad t \in \mathfrak{J}, s \in C_{R}, \tau \in \mathfrak{J}, \\
& \sum_{j \in C} a_{i j} y_{i j}^{t}
\end{aligned}
$$$$
\leq \widehat{x}_{i}+\sum_{\tau=0}^{t-1}\left(\sum_{k \in C} a_{k i} y_{k i}^{\tau}-\sum_{j \in C} a_{i j} y_{i j}^{\tau}\right),
$$$$
\forall i \in C \backslash C_{R} \cup C_{S}, t \in \mathfrak{\Im},
$$

$\sum_{k \in C} a_{k i} y_{k i}^{t}$

$$
\begin{aligned}
\leq \delta_{i}^{t}\left(N_{i}^{t}+\chi_{i} b_{i}-\widehat{x}_{i}\right. \\
\left.\quad-\sum_{\tau=0}^{t-1}\left(\sum_{k \in C} a_{k i} y_{k i}^{\tau}-\sum_{j \in C} a_{i j} y_{i j}^{\tau}\right)\right)
\end{aligned}
$$

$\forall i \in C \backslash C_{R} \cup C_{S}, t \in \mathfrak{\Im}$,

$\sum_{k \in C} a_{k i} y_{k i}^{t} \leq Q_{i}^{t}+\phi_{i} b_{i}, \quad \forall i \in C \backslash C_{R} \cup C_{S}, t \in \mathfrak{J}$,

$\sum_{j \in C} a_{i j} y_{i j}^{t} \leq Q_{i}^{t}+\phi_{i} b_{i}, \quad \forall i \in C \backslash C_{S}, t \in \mathfrak{I}$,

$y_{i j}^{0}=0, \quad \forall(i, j) \in C \times C$,

$y_{i j}^{t} \geq 0, \quad \forall(i, j) \in C \times C, t \in \mathfrak{J}$,

$\sum_{i \in C \backslash C_{s}} b_{i} \leq B$,

$b_{i} \geq 0, \quad \forall i \in C \backslash C_{S}$,

where $\mu$ is a set of dual variables and the numerical indexes are used for notational simplicity. 


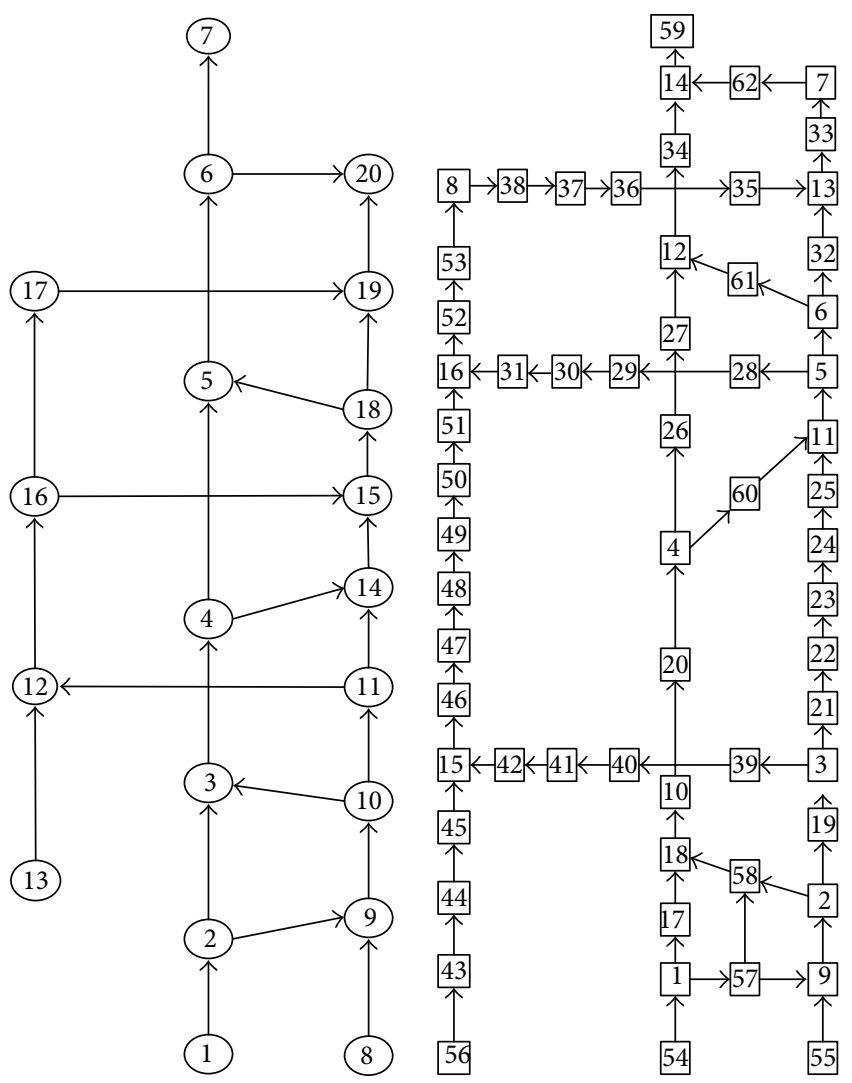

FIgURE 1: Test network [40].

TABLE 1: Characteristic of the cells.

\begin{tabular}{lcccc}
\hline Cell & Source cells & Sink cells & Freeway cells & Arterial cells \\
\hline$N_{i}^{t}$ & $\infty$ & $\infty$ & 20 & 10 \\
$Q_{i}^{t}$ & $\infty$ & $\infty$ & 12 & 3 \\
$\delta_{i}^{t}$ & 1 & 1 & 1 & 1 \\
$\widehat{x}_{i}$ & 0 & 0 & 0 & 0 \\
\hline
\end{tabular}

\section{Numerical Example}

The purpose of presenting the numerical example in this section is twofold: (1) to demonstrate the validity of SODNDPAARC1 and (2) to illustrate the flexibility of the SODNDPAARC1 by comparing it with SODNDP-RC1. One example network shown in Figure 1 is selected to test the proposed model. This network consists of 20 nodes and 25 links. The links in the center of the network are the freeway links, while the outer link and crosslink are the arterial links. The characteristics of network include length, speed limit, and jam density of the roads. By using this network information and setting 6 seconds as the length of a time interval, the corresponding cell network representation is constructed. This cell network is composed of 62 cells and 67 cell connectors. There are three source cells (cells 54, 55, and 56) and one sink cell (cell 59). The other is either freeway cell or arterial cells. Expected the sink cell 59, the other cells are considered for capacity expansion. The characteristics of the cells in the test network are shown in Table 1.
The planning horizon $T$ and cost coefficient $c_{i}^{t}$ except the one at the end of planning horizon are assumed to be 105 and 1 , respectively. $c_{i}^{T}$ is set to 10 to penalize the unmet demand. The model parameters $\delta$ and $\phi$ are assumed to be unity; that is, $\delta_{i}^{t}=\phi_{i}=1$, for all $i \in C, t \in \mathfrak{\Im}$. Travel demand is only generated at the three source cells and time 0 . The nominal demand is set to 63.6. That is, $d_{i}^{0}=63.6$, for all $i \in C_{R}$; the total demand is $D_{i}=2 \sum d_{i}^{t}$, for all $i \in C_{R}$. The SODNDP-AARC1 and SODNDP-RC1 problems are solved with GUROBI using GAMS [37] on a PC with Intel processor $2.8 \mathrm{GHz}$ and $4 \mathrm{~GB}$ of memory.

Table 2 reports the optimal solution of the SODNDP, SODNDP-AARC1, and SODNDP-RC1 models under different uncertainty levels when the available budget $B=100$. It can be seen from Table 2 that the optimal solution of the SODNDP-AARC1 model has remained unchanged under different uncertainty levels, while the optimal solution of the SODNDP-RC1 model keeps changing. It is clear that the adjustable robust capacity expansion plans are more stable. Tables 3 and 4 list the total travel cost and solution time of the SODNDP, SODNDP-AARC1, and SODNDPRC1 models under different uncertainty levels and available budgets. As shown in Tables 3 and 4, the total travel cost from the nominal solution, robust solution, and adjustable robust solution increases with the rise of uncertainty level $\theta$ when $B=100$. On the other hand, the total travel cost from the nominal solution, robust solution, and adjustable robust solution decreases with the increase of available budget $B$ when $\theta=0.5$. This is because the feasible region of all three models reduces with the rise of uncertainty level $\theta$; on the contrary, the feasible region of all three models enlarges with the increase of available budget $B$. At the same time, the optimal objective value of the SODNDP-RC1 model is far greater not only than the SODNDP model but also than the SODNDP-AARC1 model, while the optimal objective value of SODNDP-AARC1 model is only slightly greater than SODNDP model. This shows that the SODNDP-RC1 model is overly conservative, while SODNDP-AARC1 model is more flexible than the SODNDP-RC1 model. Moreover, the solution time of the SODNDP model and SODNDP$\mathrm{RC1}$ model is almost the same, and the solution time of the SODNDP-AARC1 model is longer than the other two models. It is due to the fact that the SODNDP-AARC1 model has more variables and constraints.

Figure 2 depicts the total travel cost from the nominal solution, robust solution, and adjustable solution and the relative improvement of the total travel cost of the robust solution against adjustable solution under different uncertainty levels and available budgets. The relative improvement in this paper is defined as

$$
\mathrm{RI}=\frac{z^{R}-z^{A R}}{z^{R}},
$$

where $z^{R}$ is the total travel cost from the robust solution and $z^{A R}$ is the total travel cost from the adjustable robust solution. As shown in Figure 2, the total travel cost from both the robust solution and adjustable robust solution decreases with the increase of available budget $B$ but increases with 
TABLE 2: The optimal solution of the SODNDP, SODNDP-RC1, and SODNDP-AARC1 when $B=100$.

\begin{tabular}{|c|c|c|c|c|c|c|c|c|c|c|c|}
\hline \multirow{2}{*}{$\begin{array}{l}\text { Cell } \\
\theta\end{array}$} & \multirow{2}{*}{$\begin{array}{c}\text { SODNDP } \\
0\end{array}$} & \multicolumn{10}{|c|}{ SODNDP-RC1 } \\
\hline & & 0.1 & 0.2 & 0.3 & 0.4 & 0.5 & 0.6 & 0.7 & 0.8 & 0.9 & 1.0 \\
\hline 1 & 8.883 & 8.523 & 8.163 & 7.663 & 7.048 & 7.482 & 6.489 & 6.615 & 0.72 & 0 & 0 \\
\hline 2 & 0.762 & 2.082 & 3.402 & 5.235 & 7.492 & 5.9 & 9.539 & 9.08 & 9.72 & 3.36 & 0 \\
\hline 4 & 8.883 & 8.523 & 8.163 & 7.663 & 7.048 & 7.482 & 6.489 & 6.615 & 5.44 & 0 & 0 \\
\hline 5 & 0 & 0 & 0 & 0 & 0 & 0 & 0 & 0 & 0 & 0 & 100 \\
\hline 9 & 0.762 & 2.082 & 3.402 & 5.235 & 7.492 & 5.9 & 9.539 & 9.08 & 9.72 & 3.36 & 0 \\
\hline 10 & 8.883 & 8.523 & 8.163 & 7.663 & 7.048 & 7.482 & 6.489 & 6.615 & 5.44 & 0 & 0 \\
\hline 12 & 8.883 & 8.523 & 8.163 & 7.663 & 7.048 & 7.482 & 6.489 & 6.615 & 5.44 & 0 & 0 \\
\hline 14 & 8.883 & 8.523 & 8.163 & 7.663 & 7.048 & 7.482 & 6.489 & 6.615 & 5.44 & 0 & 0 \\
\hline 17 & 8.883 & 8.523 & 8.163 & 7.663 & 7.048 & 7.482 & 6.489 & 6.615 & 0.72 & 0 & 0 \\
\hline 18 & 8.883 & 8.523 & 8.163 & 7.663 & 7.048 & 7.482 & 6.489 & 6.615 & 5.44 & 0 & 0 \\
\hline 20 & 8.883 & 8.523 & 8.163 & 7.663 & 7.048 & 7.482 & 6.489 & 6.615 & 5.44 & 0 & 0 \\
\hline 26 & 8.883 & 8.523 & 8.163 & 7.663 & 7.048 & 7.482 & 6.489 & 6.615 & 25.88 & 0 & 0 \\
\hline 27 & 8.883 & 8.523 & 8.163 & 7.663 & 7.048 & 7.482 & 6.489 & 6.615 & 5.44 & 0 & 0 \\
\hline 34 & 8.883 & 8.523 & 8.163 & 7.663 & 7.048 & 7.482 & 6.489 & 6.615 & 5.44 & 89.92 & 0 \\
\hline 58 & 0.762 & 2.082 & 3.402 & 5.235 & 7.492 & 5.9 & 9.539 & 9.08 & 9.72 & 3.36 & 0 \\
\hline Cell & & \multicolumn{10}{|c|}{ SODNDP-AARC1 } \\
\hline$\theta$ & & 0.1 & 0.2 & 0.3 & 0.4 & 0.5 & 0.6 & 0.7 & 0.8 & 0.9 & 1.0 \\
\hline 1 & & 9.091 & 9.091 & 9.091 & 9.091 & 9.091 & 9.091 & 9.091 & 9.091 & 9.091 & 9.091 \\
\hline 4 & & 9.091 & 9.091 & 9.091 & 9.091 & 9.091 & 9.091 & 9.091 & 9.091 & 9.091 & 9.091 \\
\hline 10 & & 9.091 & 9.091 & 9.091 & 9.091 & 9.091 & 9.091 & 9.091 & 9.091 & 9.091 & 9.091 \\
\hline 12 & & 9.091 & 9.091 & 9.091 & 9.091 & 9.091 & 9.091 & 9.091 & 9.091 & 9.091 & 9.091 \\
\hline 14 & & 9.091 & 9.091 & 9.091 & 9.091 & 9.091 & 9.091 & 9.091 & 9.091 & 9.091 & 9.091 \\
\hline 17 & & 9.091 & 9.091 & 9.091 & 9.091 & 9.091 & 9.091 & 9.091 & 9.091 & 9.091 & 9.091 \\
\hline 18 & & 9.091 & 9.091 & 9.091 & 9.091 & 9.091 & 9.091 & 9.091 & 9.091 & 9.091 & 9.091 \\
\hline 20 & & 9.091 & 9.091 & 9.091 & 9.091 & 9.091 & 9.091 & 9.091 & 9.091 & 9.091 & 9.091 \\
\hline 26 & & 9.091 & 9.091 & 9.091 & 9.091 & 9.091 & 9.091 & 9.091 & 9.091 & 9.091 & 9.091 \\
\hline 27 & & 9.091 & 9.091 & 9.091 & 9.091 & 9.091 & 9.091 & 9.091 & 9.091 & 9.091 & 9.091 \\
\hline 34 & & 9.091 & 9.091 & 9.091 & 9.091 & 9.091 & 9.091 & 9.091 & 9.091 & 9.091 & 9.091 \\
\hline
\end{tabular}

TABLE 3: The optimal objective and solution time of the SODNDP, SODNDP-RC1, and SODNDP-AARC1 models under different uncertainty levels when $B=100$.

\begin{tabular}{|c|c|c|c|c|c|c|}
\hline$\theta$ & SODNDP & $\begin{array}{c}\text { SODNDP } \\
\text { solution time }(\mathrm{sec})\end{array}$ & SODNDP-RC1 & $\begin{array}{c}\text { SODNDP-RC1 } \\
\text { solution time }(\mathrm{sec})\end{array}$ & SODNDP-AARC1 & $\begin{array}{l}\text { SODNDP-AARCl } \\
\text { solution time (sec) }\end{array}$ \\
\hline 0.1 & 4017.0113 & 0.08 & 16434.0362 & 0.06 & 4505.6073 & 83.09 \\
\hline 0.2 & & & 18757.4762 & 0.06 & 5001.6873 & 34.63 \\
\hline 0.3 & & & 21123.6929 & 0.06 & 5497.7673 & 65.28 \\
\hline 0.4 & & & 23525.3110 & 0.06 & 5993.8473 & 93.75 \\
\hline 0.5 & & & 26690.3091 & 0.06 & 6489.9273 & 86.87 \\
\hline 0.6 & & & 30183.6424 & 0.05 & 6986.0073 & 36.84 \\
\hline 0.7 & & & 33688.2109 & 0.06 & 7482.0873 & 50.98 \\
\hline 0.8 & & & 37206.0000 & 0.05 & 7978.1673 & 83.61 \\
\hline 0.9 & & & 40735.8000 & 0.06 & 8474.2473 & 48.67 \\
\hline 1.0 & & & 44265.6000 & 0.03 & 8970.3273 & 188.84 \\
\hline
\end{tabular}


TABLE 4: The total travel cost and runtimes of the SODNDP, SODNDP-RC1, and SODNDP-AARC1 models under different available budgets when $\theta=0.5$.

\begin{tabular}{lcccccc}
\hline$B$ & SODNDP & $\begin{array}{c}\text { SODNDP solution } \\
\text { time }(\mathrm{sec})\end{array}$ & SODNDP-RC1 & $\begin{array}{c}\text { SODNDP-RC1 } \\
\text { solution time (sec) }\end{array}$ & SODNDP-AARC1 & $\begin{array}{c}\text { SODNDP-AARC1 } \\
\text { solution time (sec) }\end{array}$ \\
\hline 10 & 4304.0727 & 0.05 & 27806.2868 & 0.06 & 6784.4727 & 25.30 \\
20 & 4271.3455 & 0.06 & 27575.3434 & 0.06 & 6751.7455 & 34.95 \\
30 & 4238.6182 & 0.06 & 27344.4000 & 0.06 & 6719.0182 & 29.41 \\
40 & 4205.8909 & 0.06 & 27113.4566 & 0.06 & 6686.2909 & 67.55 \\
50 & 4173.1636 & 0.08 & 26914.0258 & 0.05 & 6653.5636 & 26.08 \\
60 & 4140.4364 & 0.06 & 26716.6065 & 0.05 & 6620.8364 & 32.89 \\
70 & 4107.7091 & 0.06 & 26706.6727 & 0.06 & 6588.1091 & 59.81 \\
80 & 4074.9818 & 0.05 & 26701.2182 & 0.05 & 6555.3818 & 68.64 \\
90 & 4044.1811 & 0.06 & 26695.7636 & 0.06 & 6522.6545 & 79.20 \\
\hline
\end{tabular}

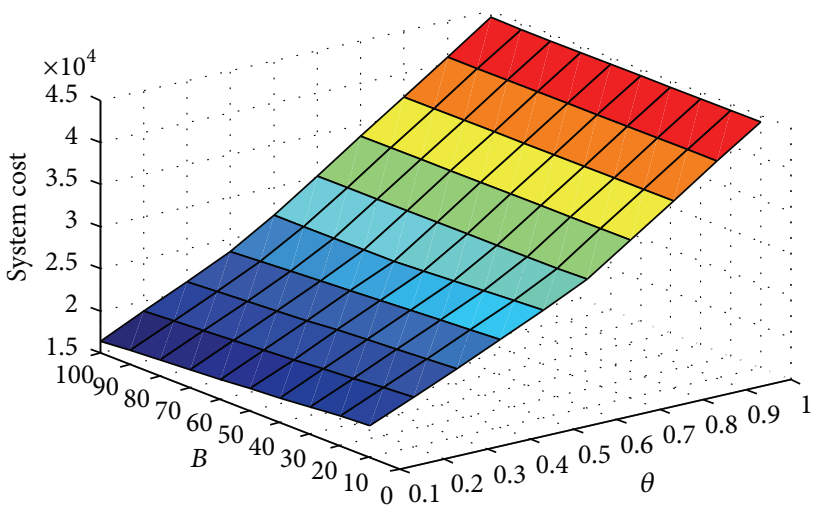

(a) The optimal travel cost of the SODNDP-RC1

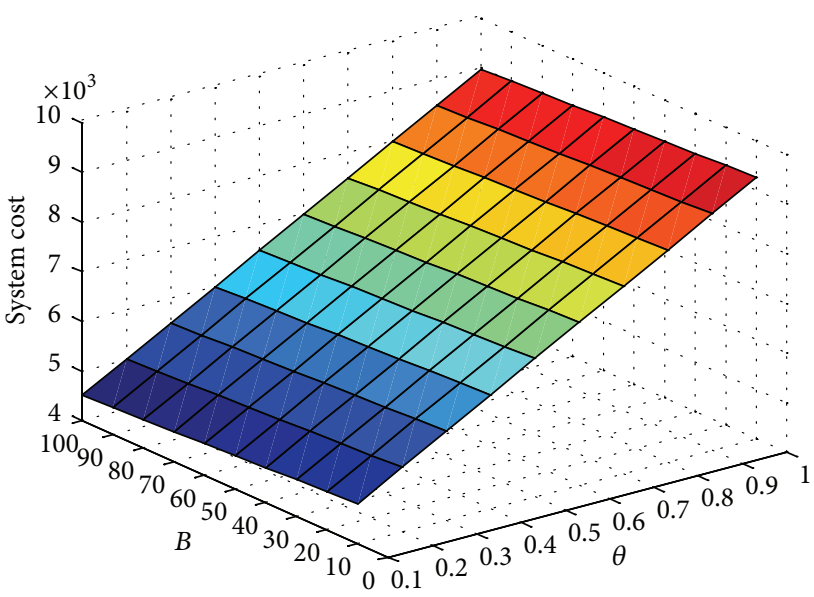

(b) The optimal travel cost of the SODNDP-AARC1

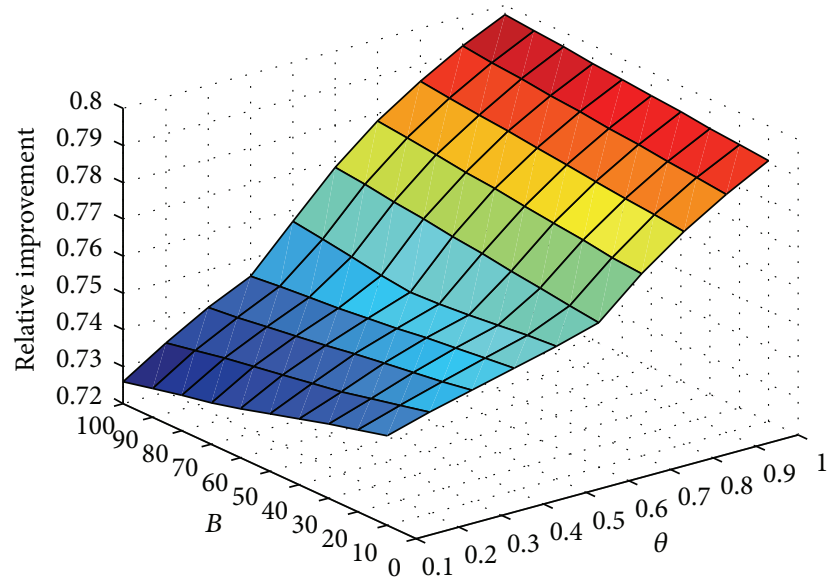

(c) The relative improvement of the optimal objective value of the SODNDP-RC1 against SODNDP-AARC1

FIGURE 2: The optimal objective value of the SODNDP-RC1 and SODNDP-AARC1 models, and the relative improvement of the optimal objective value of the SODNDP-RC1 against SODNDP-AARC1 under different available budgets and uncertainty levels. 


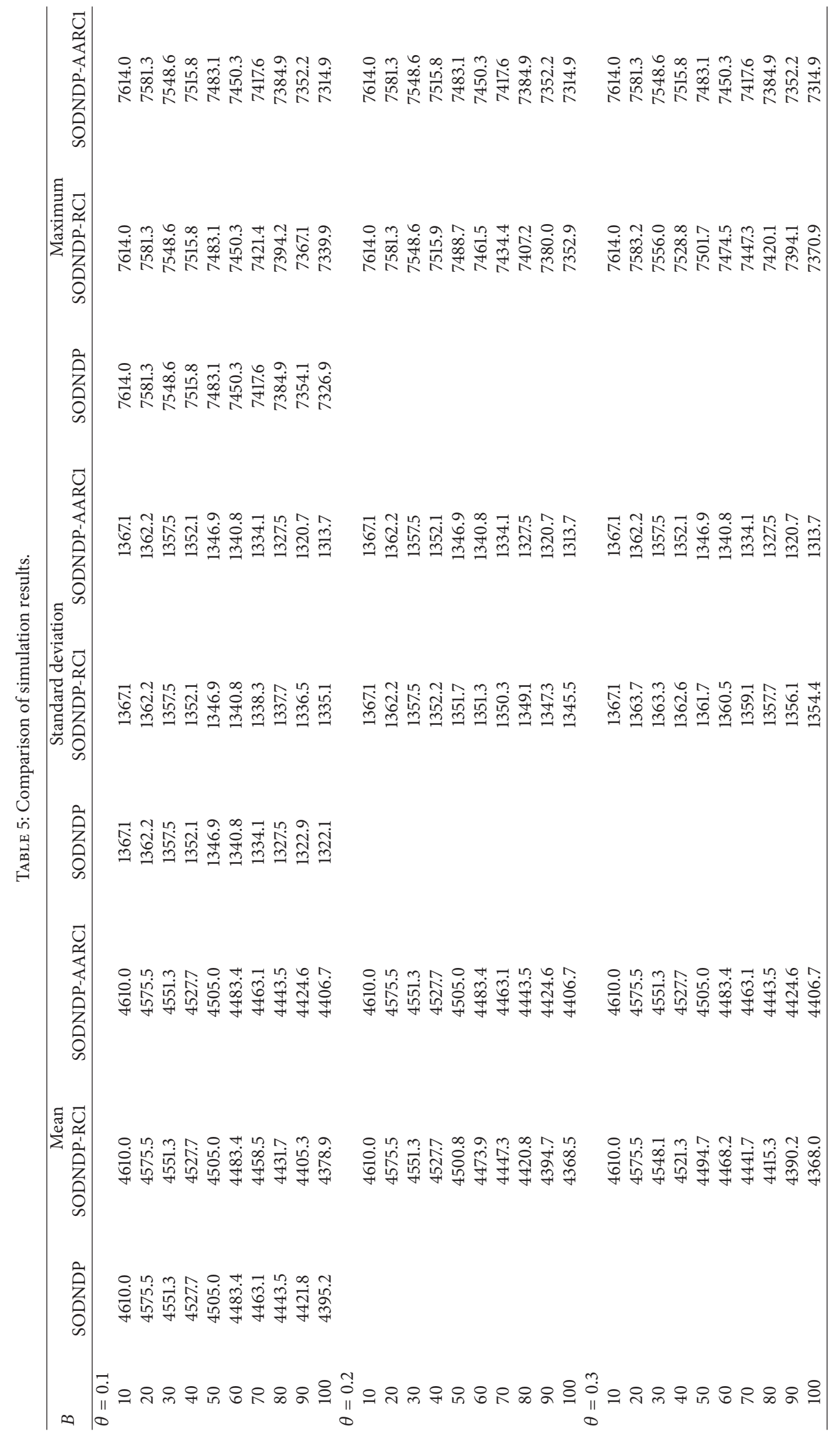


an increasing value of the uncertainty level $\theta$. However, the total travel cost from the adjustable robust solution is far lower than the robust solution under any circumstances. This also shows that the adjustable solution is less conservative than the robust solution. When the uncertainty level $\theta \geq$ 0.7 , the total travel cost from the robust solution basically remains unchanged with the increase of available budget $B$, which means that the increase of available budget has no much effect on the improvement of traffic congestion. This implies that some investments are wasted, and this case does not appear in the SODNDP-AARC1 model. Moreover, the relative improvement of the total travel cost of the robust solution against adjustable solution is monotonically decreasing function with respect to the available budget $B$ and is monotonically increasing function with respect to the uncertainty level $\theta$. When the uncertainty level $\theta \geq 0.5$, the relative improvement range of the total travel cost of the robust solution against adjustable solution becomes larger, and it almost reaches $80 \%$ when the uncertainty level $\theta$ equals 1. Thus, the SODNDP-AARC1 model is less conservative than the SODNDP-RC1 model; therefore, it becomes more attractive.

To compare the operating behaviors of the three capacity expansion plans, nominal, robust, and adjustable robust, we randomly generated 100 travel demand vectors in which the demand of each O-D pair satisfies the normal distribution whose mean is $\bar{d}_{i}^{t}$ and standard deviation is $0.5 \bar{d}_{i}^{t}$. For each random vector $d_{i}^{t}$, for all $i \in C_{R}$, the total travel cost from the nominal, robust, and adjustable robust capacity expansion plans is computed. The mean, standard deviation, and maximum of the total travel cost associated with nominal, robust, and adjustable robust capacity expansion plans are shown in Table 5 . It can be seen that the mean, standard deviation, and maximum of the total travel cost from the nominal, robust, and adjustable robust capacity expansion plans all decrease as the available budget $B$ increases. The mean and maximum of the total travel cost from the robust capacity expansion plans reduce when the uncertainty level $\theta$ increases, and standard deviation of the total travel cost from the robust capacity expansion plans increases when the uncertainty level $\theta$ increases. On the other hand, the mean, standard deviation, and maximum of the total travel cost from adjustable robust capacity expansion plans remain unchanged for all uncertainty levels. This is because the adjustable robust capacity expansion plans are the same under different uncertainty levels. In addition, the mean of the total travel cost from the adjustable robust capacity expansion plans is greater than that of the robust capacity expansion plans; however, in all case, the standard deviation and maximum of the total travel cost from the adjustable robust capacity expansion plans are less than or equal to those of the robust capacity expansion plans.

The optimal objective value of the SODNDP-RC1 and SODNDP-AARC1 models is denoted as $z^{*}\left(b^{R}\right)$ and $z^{*}\left(b^{A R}\right)$, respectively. Moreover, the optimal objective value of the SODNDP model is denoted as $z^{*}\left(b^{N}\right)$, where it is computed based on the nominal demand $d_{i}^{t}$. Among the 100 travel demand vectors $\left(d_{i}^{t}\right)$, we compare total cost from

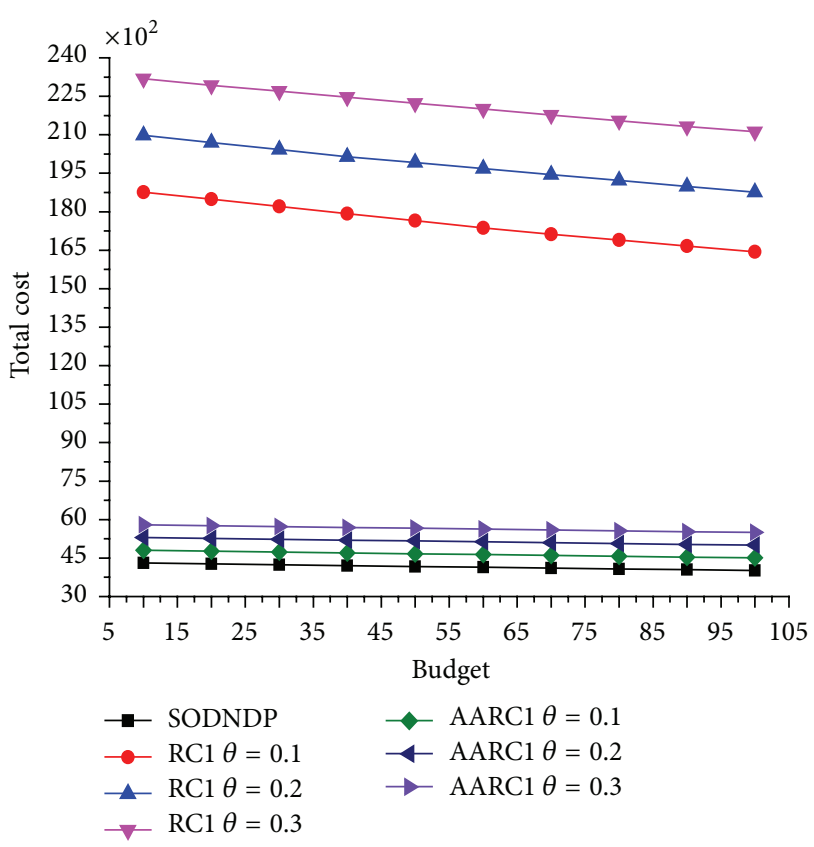

FIGURE 3: The optimal objective value of the SODNDP, SODNDP$\mathrm{RC}$, and SODNDP-AARC1 models.

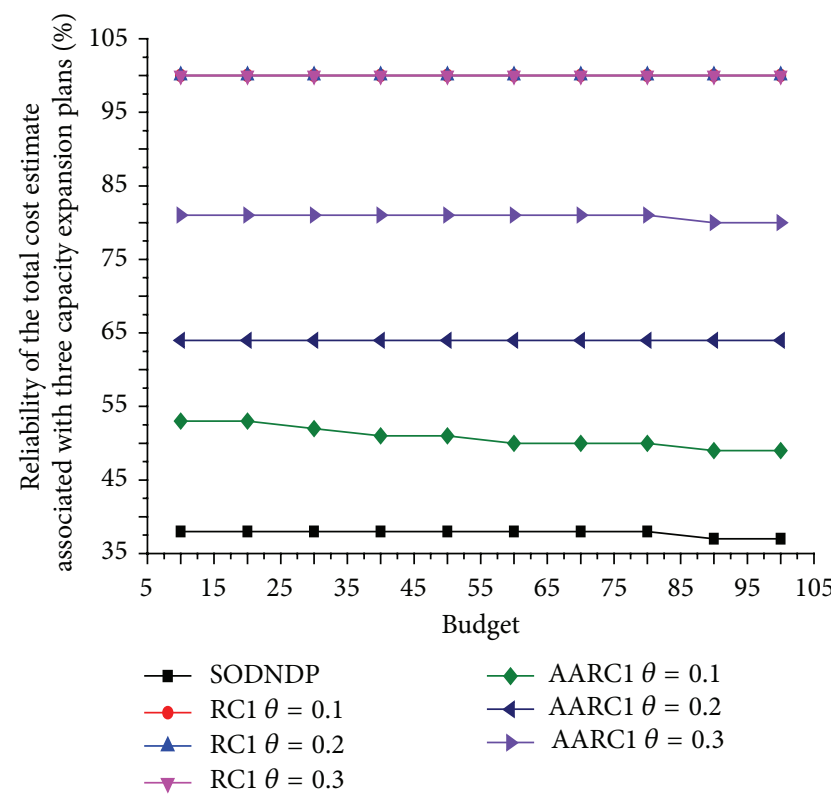

FIgURE 4: Reliability of the total cost estimate associated with nominal, robust, and adjustable robust capacity expansion plans.

the nominal, robust, and adjustable robust capacity expansion plans against $z^{*}\left(b^{N}\right), z^{*}\left(b^{R}\right)$, and $z^{*}\left(b^{A R}\right)$, respectively. Figure 3 depicts the optimal objective value of the SODNDP, SODNDP-RC1, and SODNDP-AARC1 models, $z^{*}\left(b^{N}\right)$, $z^{*}\left(b^{R}\right)$, and $z^{*}\left(b^{A R}\right)$, under different available budgets and uncertainty levels. Figure 4 shows the percentages for which $\sum_{t \in \mathfrak{J}, i \in C \backslash C_{s}} c_{i}^{t} x_{i}^{t}\left(d, b^{N}\right) \leq z^{*}\left(b^{N}\right), \sum_{t \in \mathfrak{J}, i \in C \backslash C_{s}} c_{i}^{t} x_{i}^{t}\left(d, b^{R}\right) \leq$ $z^{*}\left(b^{R}\right)$ and $\sum_{t \in \Im, i \in C \backslash C_{s}} c_{i}^{t} x_{i}^{t}\left(d, b^{A R}\right) \leq z^{*}\left(b^{A R}\right)$ under different 
available budgets and uncertainty levels. As shown in Figures 3 and 4, on average, approximately $38 \%$ or 38 random demands out of the 100 yields total travel cost no larger than $z^{*}\left(b^{N}\right)$. In other words, the nominal total travel cost is $38 \%$ reliable. One the other hand, the average reliability of the robust total travel cost is $100 \%$ under different uncertainty levels, and the average reliability of the adjustable robust total travel cost is $50 \%$ when uncertainty level $\theta=0.1$. Although the average reliability of the robust total travel cost is higher than that of the adjustable total travel cost, the robust total travel cost is far greater than the adjustable robust total travel cost, which demonstrates that the robust capacity expansion plans are overly conservative. In addition, the average reliability of the adjustable robust total travel cost is monotonically increasing with the rise of uncertainty level $\theta$. The average reliability of the adjustable robust total travel cost is $80 \%$ when uncertainty level $\theta=0.3$. The average reliability of the adjustable robust total travel cost is slightly lower than that of the robust total travel cost; however, the adjustable robust capacity expansion plans yield lower total travel cost. Thus, it is continuing to suggest that the adjustable robust capacity expansion plans are more flexible.

\section{Conclusion and Further Research}

This paper applied the adjustable robust optimization methodology to the CTM-based single-level system-optimal dynamic NDP (DNDP) under demand uncertainty. We assumed that the uncertain demand belongs to a polyhedral set and used the adjustable robust optimization methodology to formulate the AARC of DNDP. In addition, the RC of DNDP was formulated by using the usual robust optimization approach, which was compared against the AARC of DNDP. Numerical example results show that the adjustable robust capacity expansion plans are more flexible and significantly less conservative than the usual robust capacity expansion plans.

Several directions for future research are worth noting. Firstly, in this paper, the used LP based CTM model allows vehicle holding [35], which may be unrealistic. Secondly, ARO approach can be applied to alternative deterministic mathematical formulation [38] to overcome this problem. In addition, an extension to consider unbounded uncertainty set with globalized robust optimization [39] is another interesting research direction.

\section{Notations}

Sets

$\mathfrak{~}$ : Set of time intervals $\{1,2, \ldots, T\}$

$C$ : Set of cells

$C_{R}:$ Set of source cells

$C_{S}$ : Set of sink cells

A: Adjacent matrix, $A=\left\{a_{i j}\right\}$; if cell $i$ is connected to cell $j$, then $a_{i j}=1$; otherwise $a_{i j}=0$.

\section{Parameters}

$d_{i}^{t}$ : Demand generated in cell $i$ at time $t$, $i \in C_{R}$

$c_{i}^{t}$ : Travel cost in cell $i$ at time $t$

$N_{i}^{t}$ : Capacity in cell $i$ at time $t$

$\delta_{i}^{t}$ : Ratio of the free-flow speed over the back propagation speed of cell $i$ at time $t$

$Q_{i}^{t}$ : Inflow/outflow capacity of cell $i$ at time $t$

$B$ : Total investment budget

$\chi_{i}$ : Increase in $N_{i}^{t}$ for a unit increase of $b_{i}$

$\phi_{i}$ : Increase in $Q_{i}^{t}$ for a unit increase of $b_{i}$

$\widehat{x}_{i}$ : Initial number of vehicles of cell $i$.

Variables

$b_{i}$ : Investment cost spent on cell $i, b=\left(\ldots, b_{i}, \ldots\right)$

$x_{i}^{t}$ : Number of vehicles staying in cell $i$ at time $t, x=\left(\ldots, x_{i}^{t}, \ldots\right)$

$y_{i j}^{t}$ : Number of vehicles moving from cell $i$ to cell $j$ at time $t, y=\left(\ldots, y_{i j}^{t}, \ldots\right)$.

\section{Conflict of Interests}

The authors declare that there is no conflict of interests regarding the publication of this paper.

\section{Acknowledgments}

The work described in this paper was jointly supported by grants from the National Natural Science Foundation of China (71131001) and the National Basic Research Program of China (2012CB725400).

\section{References}

[1] B. D. Chung, T. Yao, C. Xie, and A. Thorsen, "Robust optimization model for a dynamic design problem under demand uncertainty," Networks and Spatial Economics, vol. 11, no. 2, pp. 371-389, 2011.

[2] S. T. Waller, J. L. Schofer, and A. K. Ziliaskopoulos, "Evaluation with traffic assignment under demand uncertainty," Transportation Research Record, no. 1771, pp. 69-74, 2001.

[3] A. Chen, Z. Zhou, P. Chootinan, S. Ryu, C. Yang, and S. C. Wong, "Transportation network design under uncertainty: a review and new developments," Transport Reviews, vol. 31, no. 6, pp. 743-768, 2011.

[4] T. L. Magnanti and R. T. Wong, "Network design and transportation planning: models and algorithms," Transportation Science, vol. 18, no. 1, pp. 1-55, 1984.

[5] M. Minoux, "Network synthesis and optimum network design problems: models, solution methods and applications," Networks, vol. 19, no. 3, pp. 313-360, 1989.

[6] H. Yang and M. G. H. Bell, "Models and algorithms for road network design: a review and some new developments," Transport Reviews, vol. 18, no. 3, pp. 257-278, 1998.

[7] D.-Y. Lin, A. Karoonsoontawong, and S. T. Waller, "A DantzigWolfe decomposition based heuristic scheme for bi-level 
dynamic network design problem," Networks and Spatial Economics, vol. 11, no. 1, pp. 101-126, 2011.

[8] K. Jeon, S. V. Ukkusuri, and S. T. Waller, "Heuristic approach for discrete network design problem accounting for dynamic traffic assignment conditions: formulation, solution methodologies, implementations and computation experiences," in Proceedings of the 84th Annual Meeting of the Transportation Research Board, Washington, DC, USA, 2005.

[9] S. T. Waller, K. C. Mouskos, D. Kamaryiannis, and A. K. Ziliaskopoulos, "A linear model for the continuous network design problem," Computer-Aided Civil and Infrastructure Engineering, vol. 21, no. 5, pp. 334-345, 2006.

[10] S. V. Ukkusuri and S. T. Waller, "Linear programming models for the user and system optimal dynamic network design problem: formulations, comparisons and extensions," Networks and Spatial Economics, vol. 8, no. 4, pp. 383-406, 2008.

[11] D.-Y. Lin, "A dual variable approximation-based descent method for a bi-level continuous dynamic network design problem," Computer-Aided Civil and Infrastructure Engineering, vol. 26, no. 8, pp. 581-594, 2011.

[12] B. N. Janson, "Network design effects of dynamic traffic assignment," Journal of Transportation Engineering, vol. 121, no. 1, pp. 1-13, 1995.

[13] S. T. Waller, Optimization and control of stochastic dynamic transportation system: formulations, solution methodologies, and computational experience [Ph.D. dissertation], Northwestern University, 2000.

[14] S. T. Waller and A. K. Ziliaskopoulos, "Stochastic dynamic network design problem," Transportation Research Record, no. 1771, pp. 106-113, 2001.

[15] A. Karoonsoontawong and S. T. Waller, "Comparison of systemand user-optimal stochastic dynamic network design models using Monte Carlo bounding techniques," Transportation Research Record, vol. 1923, pp. 91-102, 2005.

[16] A. Karoonsoontawong and S. T. Waller, "Dynamic continuous network design problem: linear bilevel programming and metaheuristic approaches," Transportation Research Record, no. 1964, pp. 104-117, 2006.

[17] A. Karoonsoontawong and S. T. Waller, "Robust dynamic continuous network design problem," Transportation Research Record, no. 2029, pp. 58-71, 2007.

[18] A. Karoonsoontawong and S. T. Waller, "Integrated network capacity expansion and traffic signal optimization problem: robust bi-level dynamic formulation," Networks and Spatial Economics, vol. 10, no. 4, pp. 525-550, 2010.

[19] C. F. Daganzo, "The cell transmission model: a dynamic representation of highway traffic consistent with the hydrodynamic theory," Transportation Research Part B, vol. 28, no. 4, pp. 269287, 1994.

[20] C. F. Daganzo, "The cell transmission model-part II: network traffic," Transportation Research Part B, vol. 29, no. 2, pp. 79-93, 1995.

[21] A. K. Ziliaskopoulos, "Linear programming model for the single destination System Optimum Dynamic Traffic Assignment problem," Transportation Science, vol. 34, no. 1, pp. 37-49, 2000.

[22] A. Ben-Tal, A. Goryashko, E. Guslitzer, and A. Nemirovski, "Adjustable robust solutions of uncertain linear programs," Mathematical Programming. A Publication of the Mathematical Programming Society, vol. 99, no. 2, pp. 351-376, 2004.

[23] A. Ben-Tal and A. Nemirovski, "Robust convex optimization," Mathematics of Operations Research, vol. 23, no. 4, pp. 769-805, 1998.
[24] A. Ben-Tal and A. Nemirovski, "Robust solutions of uncertain linear programs," Operations Research Letters, vol. 25, no. 1, pp. $1-13,1999$.

[25] A. Ben-Tal and A. Nemirovski, "Robust solutions of linear programming problems contaminated with uncertain data," Mathematical Programming, vol. 88, no. 3, pp. 411-424, 2000.

[26] A. Ben-Tal and A. Nemirovski, "Robust optimizationmethodology and applications," Mathematical Programming, vol. 92, no. 3, pp. 453-480, 2002.

[27] D. Bertsimas and M. Sim, “The price of robustness," Operations Research, vol. 52, no. 1, pp. 35-53, 2004.

[28] J. M. Mulvey, R. J. Vanderbei, and S. A. Zenios, "Robust optimization of large-scale systems," Operations Research, vol. 43, no. 2, pp. 264-281, 1995.

[29] Y. Yin and S. Lawphongpanich, "A robust approach to continuous network design problems with demand uncertainty," in Proceedings of 17th International Symposium of Transportation and Traffic Theory, R. E. Allsop, M. G. H. Bell, and B. G. Heydecker, Eds., pp. 111-126, 2007.

[30] Y. Yin, S. M. Madanat, and X.-Y. Lu, "Robust improvement schemes for road networks under demand uncertainty," European Journal of Operational Research, vol. 198, no. 2, pp. 470479, 2009.

[31] Y. Lou, Y. Yin, and S. Lawphongpanich, "Robust approach to discrete network designs with demand uncertainty," Transportation Research Record, no. 2090, pp. 86-94, 2009.

[32] T. Yao, S. R. Mandala, and B. D. Chung, "Evacuation transportation planning under uncertainty: a robust optimization approach," Networks and Spatial Economics, vol. 9, no. 2, pp. 171189, 2009.

[33] B. D. Chung, T. Yao, T. L. Friesz, and H. Liu, "Dynamic congestion pricing with demand uncertainty: a robust optimization approach," Transportation Research Part B, vol. 46, no. 10, pp. 1504-1518, 2012.

[34] A. Ben-Tal, B. D. Chung, S. R. Mandala, and T. Yao, "Robust optimization for emergency logistics planning: risk mitigation in humanitarian relief supply chains," Transportation Research Part B, vol. 45, no. 8, pp. 1177-1189, 2011.

[35] K. Doan and S. V. Ukkusuri, "On the holding back problem in the cell transmission based dynamic traffic assignment models," Transportation Research Part B, vol. 46, no. 9, pp. 1218-1238.

[36] S. Travis Waller and A. K. Ziliaskopoulos, "A chanceconstrained based stochastic dynamic traffic assignment model: analysis, formulation and solution algorithms," Transportation Research C, vol. 14, no. 6, pp. 418-427, 2006.

[37] A. Brooke, D. Kendirick, and A. Meeraus, GAMS: A USer's Guide, The Scientific Press, San Franciso, Calif, USA, 1992.

[38] F. Zhu and S. V. Ukkusuri, "A cell based dynamic system optimum model with non-holding back flows," Transportation Research Part C, vol. 36, pp. 367-380, 2013.

[39] A. Ben-Tal, S. Boyd, and A. Nemirovski, "Extending scope of robust optimization: comprehensive robust counterparts of uncertain problems," Mathematical Programming, vol. 107, no. 1-2, pp. 63-89, 2006.

[40] Y. Li, S. T. Waller, and T. Ziliaskopoulos, "A decomposition scheme for system optimal dynamic traffic assignment models," Network Spatial Economic, vol. 3, no. 4, pp. 441-455, 2003. 


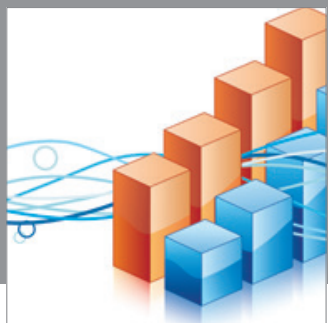

Advances in

Operations Research

mansans

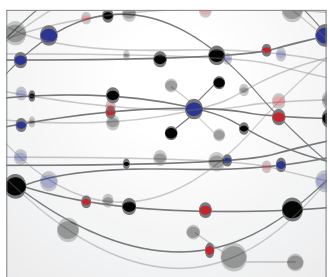

The Scientific World Journal
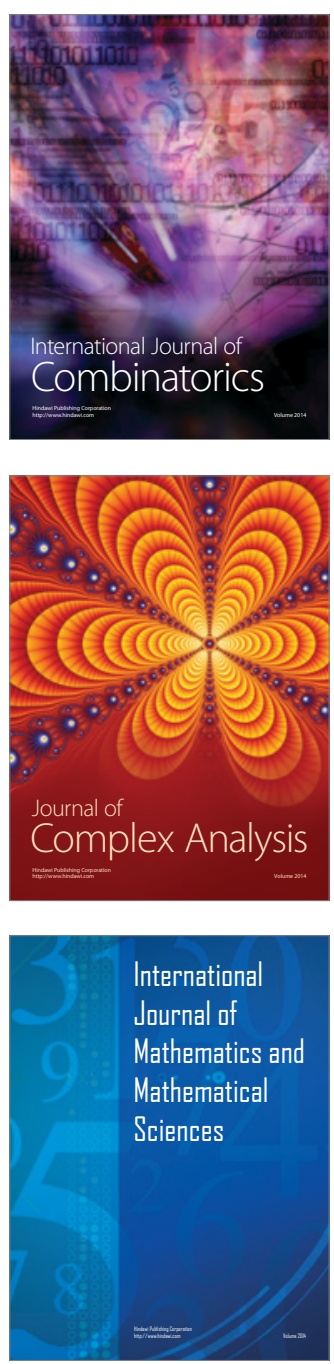
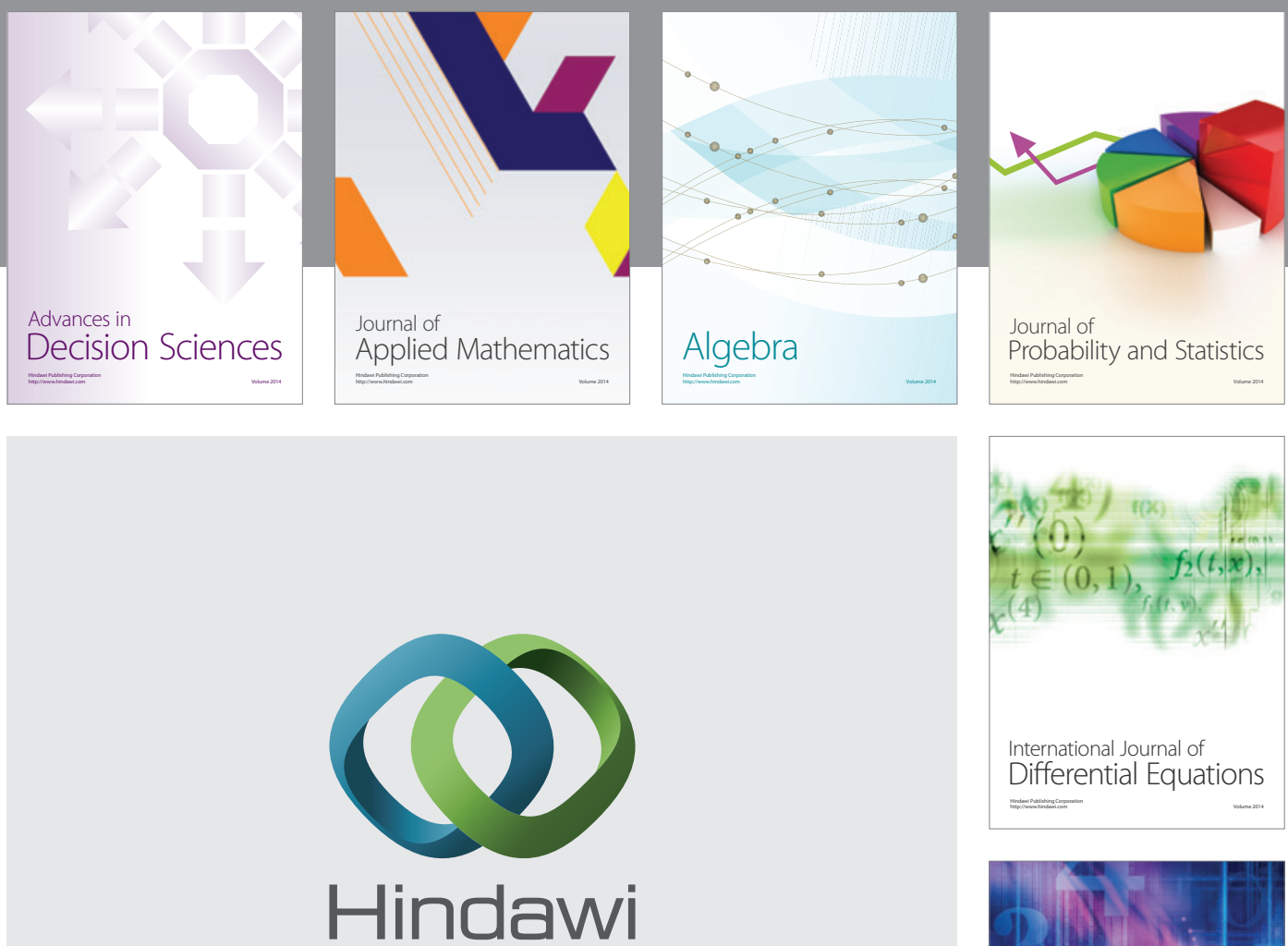

Submit your manuscripts at http://www.hindawi.com
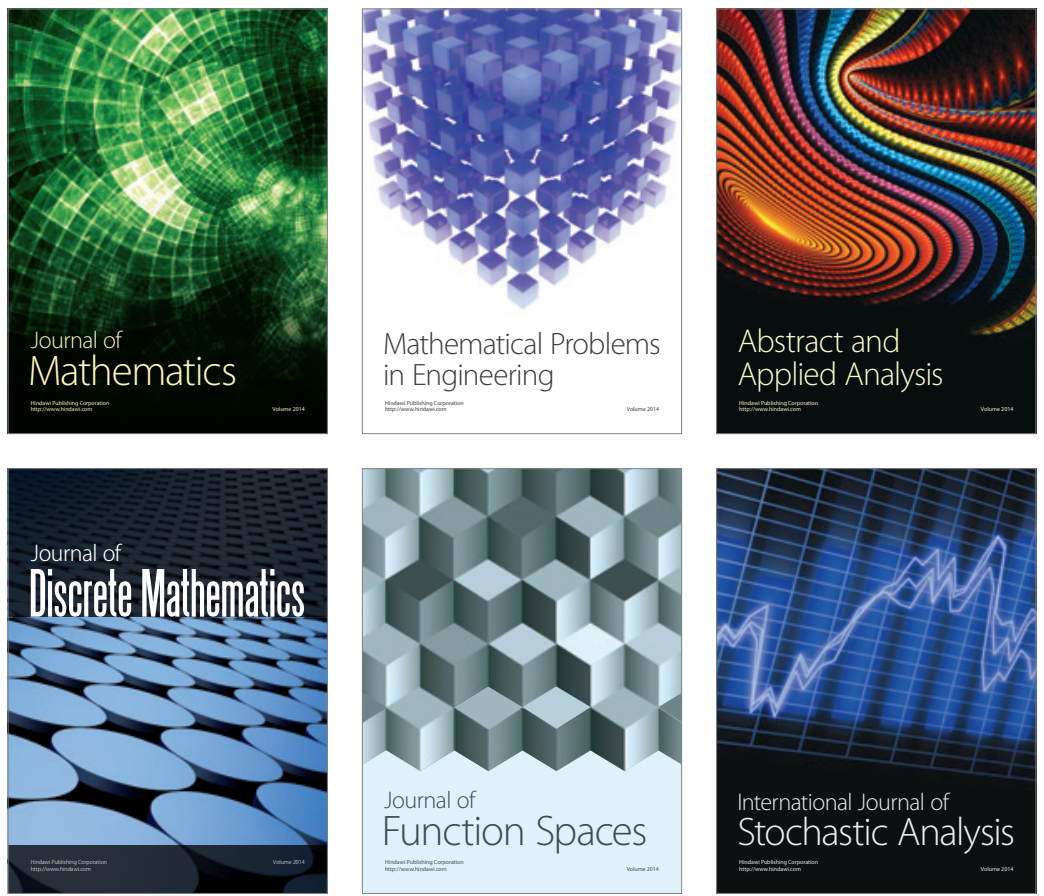

Journal of

Function Spaces

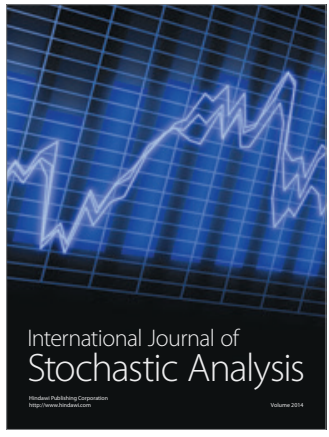

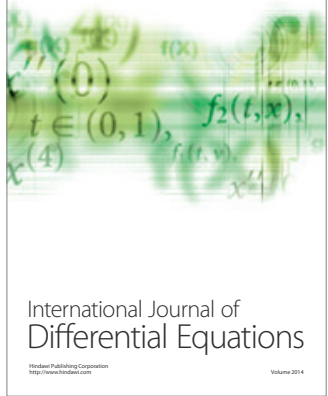
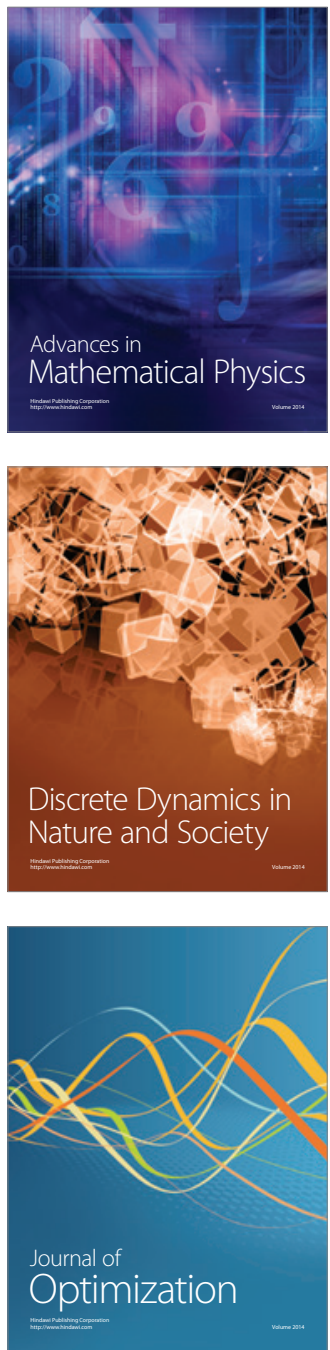